\title{
Reactive Fluid Flow Models and Applications to Diagenesis, Mineral Deposits and Crustal Rocks
}

\begin{abstract}
Funds are requested for a combined theoretical and field study of coupled fluid flow, heat and mass transport, and chemical reaction in hydrothermal and metamorphic systems. An existing computer code developed by the applicants which numerically treats multi-component, finite-rate reactions combined with advective and dispersive transport in one and two dimensions and which incorporates isotopic exchange and heat and mass transfer will continue to be developed and applied in a variety of geological settings. The code we have developed simultaneously solves for mass transport and reaction, thus offering a significant improvement in computational efficiency over existing 'batch' reaction path codes. By coupling flow and chemical reaction in a hydrothermal system, we can explicitly investigate the extent to which characteristic flow-reaction paths govern the chemical evolution of the fluids in a hydrothermal system. The concept of a flow-reaction path is particularly important where certain portions of mature hydrothermal systems may exhaust the buffer capacity of the rock as the primary mineralogy is consumed. In these instances, fluids traversing distinct regions within the hydrothermal system may experience very different reaction histories, even where the system can be described as nearly isothermal.

The study of paleo-hydrothermal systems can yield some important insights into the chemical dynamics of hydrothermal systems in general. As an example of a paleohydrothermal system, we have considered the geochemical evolution of 'porphyry-copper' type mineralization as meteoric fluids encroach on early high temperature copper mineralization and alteration assemblages. As a field example, we simulated the hydrothermal system which formed the Butte Main-Stage copper-zinc veins, where early, hightemperature mineralization is redistributed by meteoric waters along through-going fractures. Current studies are extending this work to modeling of the lateritic environment in the Pijiguaos deposit of Venezuela, to the sedimentary-hosted $\mathrm{Pb}-\mathrm{Zn}-\mathrm{Ba}$ deposits in Ireland, to the metamorphic veins of the Wepawaug metamorphic complex in South-central Connecticut and to the role of fluids in the formation of isograds around the Marysville stock, Montana. In addition, we plan to now pursue the reactive flow modeling of the high-grade metamorphic complex at Naxos, Greece. Preliminary data from the complex suggests that isotopic reaction fronts are present where metamorphic fluids have moved from one lithologically and isotopically distinct unit to another. The extent of penetration of the isotopic reaction fronts into a unit may constrain the integrated fluid flux through the contact. The detailed shape of the front may provide constraints on the instanstaneous ratio of the isotopic exchange rate to the fluid flux.

Some isotopic experiments with gibbsite exchange are also proposed to better understand the use of stable isotope data in bauxitic and lateritic environments (e.g. our work on Pijiguaos, Venezuela). Furthermore, our extensive current efforts developing $a b$ initio approaches to the quantification of surface chemical reactions as well as water-rock kinetics and our experimental efforts to decipher for the first time the dependencies of rate laws on the deviation from equilibrium of various important silicates are both closely linked to the various flow models being developed and to the applications.
\end{abstract}




\section{Introduction}

A major objective of the current research on water-rock interaction is to develop a more quantitative basis for its study. The study of mass and heat transfer is central to an understanding of the chemical evolution of sedimentary basins, the migration and entrapment of hydrocarbons, the dynamics of geothermal systems, the formation of mineral deposits, and the containment of radioactive wastes. Most geochemical models which have been developed to date apply to open or closed batch systems in which the fluid composition evolves along a single path in composition space. Mathematically, these models correspond to solving a set of ordinary differential equations in which the independent variable is time or the reaction progress variable. Although these models can be extended with some approximations to the case of pure advective flow, they don't explicitly include either diffusive or dispersive mass transfer nor do they easily account for spatial variations in such important rock properties as mineral surface areas and permeability. A complete treatment of the problem of reactive flow, therefore, requires solving a set of partial differential equations in which mass transfer and chemical reaction are directly coupled.

While it can be argued that a quantitative approach to problems in the earth sciences is always preferable to a qualitative one, one can cite some problems where a quantitative approach is essential. One such problem is the disposal of toxic wastes where the ability to predict quantitatively the fate of contaminants in the subsurface will affect the safety of future generations. One aspect of a truly quantitative approach is to consider individual phenomena taking place in the rock in the context of the dynamical whole, that is, how one process couples to another. This is particularly true in problems like the disposal of nuclear wastes where the behavior of the contaminants needs to be understood over geological periods of time. Because the toxicity of the nuclear wastes may be long-lived, there is a need to consider how, for example, chemical reactions might change such rock properties as the porosity/permeability distribution adjacent to the contaminant sites. In these cases, the usual assumption made by hydrologists that the physical properties of the porous medium are constant, may not be justified. Similarly, geochemists need to recognize that the rates of most mineral-water reactions taking place in open systems can only be predicted if solute transport is explictly considered.

Although much remains to be done to achieve a rigorously quantitative treatment of water-rock interaction, we would argue that it is important to begin to understand how some of the various dynamical processes which take place in rocks couple to each other, even if the analysis is carried out on somewhat simplified variants of the actual waste sites.

In recent years significant progress has been made in formulating the theoretical basis for the study of reactive flow in geochemistry (e.g, Lasaga, 1984, 1989a and b; Lichtner, 1985 and 1988; Lichtner et al., 1986a and b; Ortoleva et al., 1987a and b). A number of excellent studies have examined the problem of hydrodynamic flow and heat transfer in various geological settings (e.g., Donaldson, 1962; Elder, 1965; Elder, 1967a and 1967b; Combarnous and Bories, 1975; Ribando and Torrance, 1976; Norton and Knight, 1977; Cathles, 1977; Cheng, 1978; Norton, 1978; Norton and Cathles, 1979; Norton and Taylor, 1979; Elder, 1981; Cathles, 1983; Norton, 1984; Garven and Freeze, 1984a and 1984b; Bethke, 1985 and 1986; Garven, 1989; Lowell, 1991). A separate line of research has focused on developing and applying quantitative models for irreversible chemical reactions in multi-component and multi-mineralic systems, beginning with the seminal studies by Helgeson (1968) and Helgeson and others (1969) of the irreversible weathering of granite. 
These early studies were followed by the application of similar models to a number of different geological environments (e.g., Brimhall, 1980; Reed, 1982; Reed, 1983; Brimhall and Ghiorso, 1983; Sverjensky, 1984; Bowers and Taylor, 1985). The logical next step in the development of quantitative models is to couple chemical reactions with mass transport (e.g., Rubin, 1983; Walsh and others, 1984; Lasaga, 1984; Lichtner, 1985, 1.988, 1992; Bryant and others, 1987; Schecter and others, 1987; Kirkner and Reeves, 1988; Ague and Brimhall, 1989; Liu and Narasimhan, 1989a and 1989b; Novak and others, 1989; Steefel and Lasaga, 1990, 1992; Steefel and Van Cappellen, 1990; Yeh and Tripathi, 1991; Wells and Ghiorso, 1991; Phillips, 1991, Lichtner and Biino, 1992). In contrast to the growing number of workers carrying out quantitative analyses of geochemical reactions and transport, 2 far smaller number of researchers have investigated how reactions and fluid flow couple through reaction-induced porosity and permeability changes](Ortoleva and others, 1987; Hoefner and Fogler, 1988; Sanford and Konikow, 1989; Steefel and Lasaga, 1990; Chen and others, 1990). The treatment of chemical reaction and fluid flow as coupled processes has not been considered feasible because of the sheer size and to some extent the complexity of the problem. Analytical solutions to the reactiontransport equation (e.g., Ogata and Banks, 1961; Bear, 1972; Fletcher and Hoffman, 1974; Bear, 1979; Bickle and McKenzie, 1988; Lassey and Blattner, 1988; Lichtner, 1988; Lasaga, 1989; Phillips, 1991) offer some important insights into the underlying dynamics of the coupled system, but because they are restricted to one-dimensional systems with a constant flow field and to a single chemical component, they can't describe the full range of complex phenomena which emerge when multi-component, multi-dimensional transport and reaction are considered. [In their full form, the governing differential equations for coupled reaction and transport are nonlinear and therefore must be solved using numerical methods. The dramatic increase in computational power which has become available in the last few years at relatively low cost has made the numerical treatment of the problem of multi-dimensional and multi-component reaction and transport feasible for the first time. We make the further prediction that the availability of massively parallel computers in the future will eventually make the multi-dimensional reaction-transport problem routine.

One important feature is the use of a finite difference formulation for computing coupled reaction and transport for multi-component and multi-mineralic systems. The finite difference method, although computationally more intensive than the simpler reaction path models, allows for modeling of the entire range of both transient and steady-state phenomena, including advective, dispersive, and diffusive transport or any combination of these. A second important feature is the use of a kinetic formulation for all of the mineralwater reactions. The approach described is the only one we are aware of which combines a finite difference approach and kinetically-controlled mineral-water reactions. A chief innovation is the use of a global implicit or one--step method to solve the combined reaction and transport terms (i.e., the reaction and transport terms are solved simultaneously rather than in sequence). Another notable feature is the use of basis switching (rewriting the reactions locally in terms of the dominant aqueous species) which may improve both the convergence properties and the robustness of the numerical calculations. As an application, we show how the coupled reaction-transport equations can be combined with the appropriate equations for heat transfer and hydrodynamic flow in order to give a description of the coupled processes taking place in a hydrothermal system. The simulations are used to investigate the effect of reaction-induced porosity and permeability changes on the character of hydrothermal convection cells.

An application of the coupled flow and reaction code is now being developed to understand the details of the huge bauxite deposit developed or. top of the Precambrian 
Parguaza granite in the Pijiguaos district of Venezuela. The kinetic and thermodynamic data for silica, gibbsite, kaolinite and feldspar has been the center of the experimental work carried out as part of our project at Yale. These data are now being used in the fluid flow models to unravel the effects of the relative rates, the flow regimes and the non-linear nature of the rates on the observed three-dimensional structure of the deposit.

Over the past decade, we have carried out an intensive petrologic, chemical isotopic, and paragenetic study of the $\mathrm{Zn}-\mathrm{Pb}-\mathrm{Ba}$ deposit of the central Irish midlands. Caulfield et al. (1984a), Caulfield et al. (1984b), Caulfield et al.(1985b), Caulfield et al.(1986), LeHuray et al.(1986), LeHuray et al.(1987), Caulfield (ms,1987), Dixon et al., (1990; 1991), and Dixon 1993. We are now in a position to fully exploit the coupled flow and reaction code to test the validity of the conceptual model that we have developed.

A third field application of the reactive flow modeling will be to the high-grade metamorphic complex at Naxos, Greece. Prelimary data on the complex (Rye et al., 1976). suggests that isotopic reaction fronts may be present where metamorphic fluids pass from one unit to another lithologically and isotopically distinct unit. The total distance that the reaction front has migrated in from the lithologic contact (e.g., between a meta-pelite and a marble) may provide some constraints on the total, integrated fluid flux. The detailed shape of the reaction front may constrain the instantaneous ratio of the fluid flux to the exchange rate (Bickle and McKenzie, 1987).

\section{Previous Work}

A number of papers and $\mathrm{PhD}$ theses have come out recently as a result of the earlier support by this grant.

Burch, T.E., Nagy, K.L., and Lasaga, A.C., 1993, Free energy dependence of albite dissolution kinetics at $80 \times \mathrm{C}$ and $\mathrm{pH}$ 8.8: Chemical Geology, v. 175, in press

Lasaga, A.C., and Rye, D.M., 1993, Fluid flow and chemical reaction kinetics in metamorphic systems: Am. Jour. Sci., v. 293, May issue 44 pages.

Nagy, K.L., and Blum A.E., and Lasaga, A.C., 1991, Dissolution and precipitation kinetics of kaolinite at $80 \mathrm{xC}$ and $\mathrm{pH}$ 3: The dependence on solution saturation state: Am. Jour. Sci., v. 291, p. 649-686.

Nagy, K.L., and Lasaga, A.C., 1992, Dissolution and precipitation kinetics oi gibbsite at $80 \times \mathrm{C}$ and $\mathrm{pH}$ 3: The dependence on solution saturation state: Geochim. Cosmochim Acta, v. 56, p. 3093-3111.

Palin, J.M., 1992, Petrologic and Stable isotopic studies of the Wepawaug schist, Connecticut, Unpublished Ph.D. Thesis, Yale University, New Haven, Connecticut.

Steefel, C.I., and Lasaga, A.C., 1992, Putting transport into water-rock interaction models: Geology, v. 20, p. 686-684.

Steefel, C.I., and Lasaga, A.C., 1993, A model for coupled multi- component reactions and multi-dimensional fluid flow with application to permeability change in hydrothermal systems: Am. Jour. Sci., v. 293 in press.

Steefel, Carl Iver, 1992, Coupled fluid flow and chemical reaction: Model development and application to water-rock interaction, Unpublished Ph.D. Thesis, Yale University, New Haven, Connecticut. 


\subsection{Mass Transfer and Kinetic Rate Laws}

\section{Kinetic versus Equilibrium Models}

An important consideration in constructing a model for coupled reaction and transport is whether to assume that the fluid phase is in local equilibrium with the minerals in the rock or whether to use a kinetic formulation. In the case of reactions taking place between aqueous species (homogeneous reactions), most of them are much more rapid than the normal rates of transport due to fluid flow. This may not be true for various oxidationreduction reactions at low temperature (e.g.; Ohmoto and Lasaga, 1982). A number of mineral-water reactions, however, are extremely slow at low temperature (e.g., Rimstidt and Barnes, 1980; Lasaga, 1984; Nagy and others, 1991) and the assumption of local equilibrium may not be justified (Steefel and Van Cappellen, 1990; Nagy and others, 1990) (see later).

Geochemists have traditionally used activity-activity diagrarns (or stability diagrams) to represent the evolution of the chemistry of the solutions in weathering or alteration processes (e.g. Garrels and Christ, 1965; Drever, 1988). If equilibrium is always assumed between any coexisting mineral phases, then a set of reactions can be written down interconverting any pair of minerals. For example, in the weathering of a granite, the dominant reaction would be the dissolution of the feldspar:

$$
\mathrm{KAlSi}_{3} \mathrm{O}_{8}+4 \mathrm{H}^{+}+4 \mathrm{H}_{2} \mathrm{O} \rightarrow \mathrm{K}^{+}+\mathrm{Al}^{3+}+3 \mathrm{H}_{4} \mathrm{SiO}_{4}
$$

During the dissolution process, several alteration products can appear. For exarnple, we can consider gibbsite, kaolinite and mica as alteration products. The usual equilibrium relations can be written between feldspar and the alteration products:

$$
\begin{aligned}
& \mathrm{Al}_{2} \mathrm{Si}_{2} \mathrm{O}_{5}(\mathrm{OH})_{4}+5 \mathrm{H}_{2} \mathrm{O} \Longrightarrow 2 \mathrm{Al}(\mathrm{OH})_{3}+2 \mathrm{H}_{4} \mathrm{SiO}_{4} \\
& \text { kaolinite } \\
& \text { gibbsite }
\end{aligned}
$$

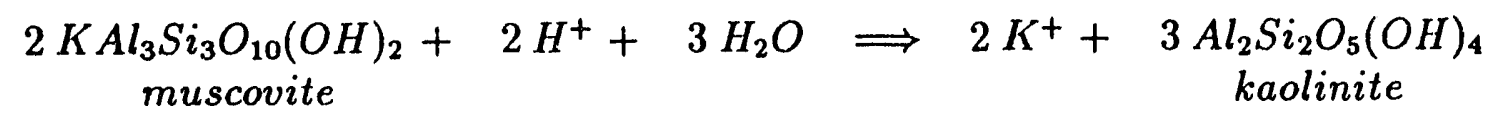

$$
\begin{aligned}
& \underset{\mathrm{K}-\text { feldspar }}{2 \mathrm{KAlSi}_{3} \mathrm{O}_{8}+2 \mathrm{H}^{+}+9 \mathrm{H}_{2} \mathrm{O}} \Longrightarrow 2 \mathrm{~K}^{+}+4 \mathrm{H}_{4} \mathrm{SiO}_{4}+\begin{array}{c}
\mathrm{Al}_{2} \mathrm{Si}_{2} \mathrm{O}_{5}(\mathrm{OH})_{4} \\
\text { kaolinite }
\end{array} \\
& \mathrm{KAl}_{3} \mathrm{Si}_{3} \mathrm{O}_{10}(\mathrm{OH})_{2}+\mathrm{H}^{+}+9 \mathrm{H}_{2} \mathrm{O} \Longrightarrow \mathrm{K}^{+}+3 \mathrm{H}_{4} \mathrm{SiO}_{4} \quad 3 \mathrm{Al}(\mathrm{OH})_{3} \\
& \text { muscovite } \\
& \begin{array}{c}
3 \mathrm{KAlSi}_{3} \mathrm{O}_{8}+2 \mathrm{H}^{+}+12 \mathrm{H}_{2} \mathrm{O} \\
\mathrm{K} \text {-feldspar }
\end{array} \Longrightarrow 2 \mathrm{~K}^{+}+6 \mathrm{H}_{4} \mathrm{SiO}_{4}+\underset{\mathrm{KAl}_{3} \mathrm{Si}_{3} \mathrm{O}_{10}(\mathrm{OH})_{2}}{\text { muscovite }}
\end{aligned}
$$

These equilibria lead to the usual activity-activity stability diagrams, which assunie that equilibrium between new phases and solutions are all achieved sufficiently fast. These stability diagrams have been used quite often in the geochemical literature. One can represent the evolution of a solution interacting with a granite, as discussed above, using the stability diagrams. Figure 1 shows a typical mass transfer equilibrium calculation. In the example, the initial $\mathrm{pH}$ was taken as $4.4, \mathrm{~T}=25 \mathrm{C}$ and the solution has initially no silica, aluminum or potassium. Such a solution could represent a typical meteoric solution. Figure $1 \mathrm{~b}$, exhibits the variation in $\mathrm{pH}$ as a function of the progress variable. 

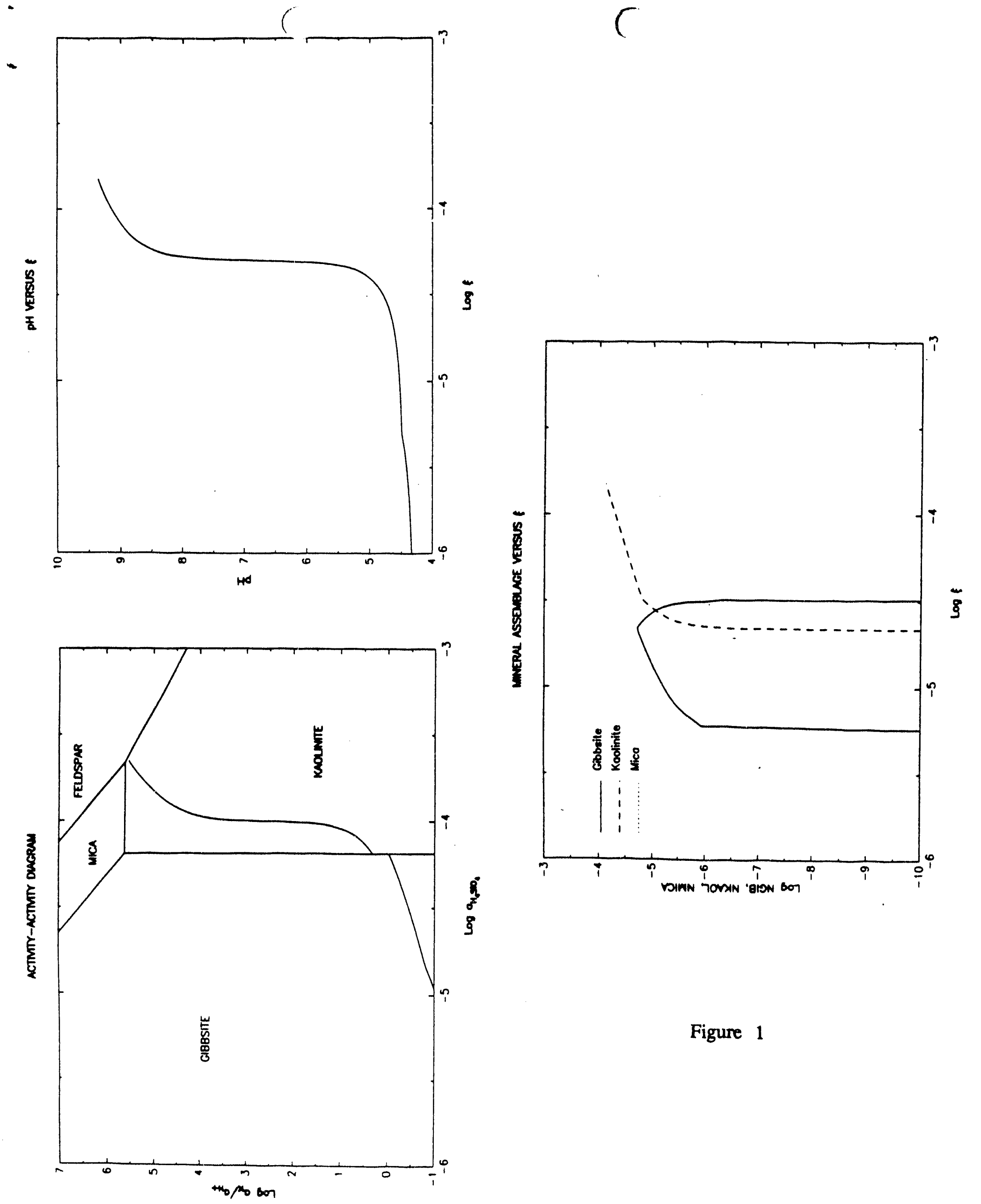

Figure 1 
Once the gibbsite begins to disappear, the $\mathrm{pH}$ increases rapidly to alkaline values reflecting the uptake of $\mathrm{H}^{+}$in reaction (1). Figure 1c, shows the variation in the product mineral phases as a function of the progress variable. As expected, gibbsite increases early on and is later replaced by kaolinite.

Ideally, one would like to apply results such as in figure 1c, to field observations of alteration or weathering fronts. For example, the bauxitization of a granite (see later discussion on the Pijiguaos bauxite deposit) can be reduced to figure 2. There are two different variables in the field situation namely, time and space. Therefore, the mass transfer calculations must relate, if possible, the progress variable to both time and space. It is precisely these type of calculations that our full flow code can address. Nonetheless, an important result can be obtained from a simplified treatment of the flow. For example, if transport is dominated by advection and the flow rate is one-dimensional (e.g. vertical) and constant and equal to $v$, then depth, $x$, and time are related by:

$$
x=v t
$$

(Note that our recent work shows that the effects of multi-dimensional flow as well as the mixing properties of dispersion are very important and must eventually be added also!). Imagine the situation whereby a homogeneous granite is being weathered by percolating solutions. The mass transfer calculation is actually following a pocket of water from its initial contact with the granite at the top. As the pocket of water percolates downward it continues to dissolve feldspar and hence to increase the progress variable of reaction (1). Note that the major difference between a dynamic and a static system is that the dissolving feldspar in the former is not the same grain during the progress of the reaction. If the rate of dissolution of the feldspar is given by

$$
\frac{d \xi}{d t}=k_{+}
$$

then it is clear that

$$
\xi=k_{+} t=\frac{k_{+}}{v} x
$$

Hence, in this simple case, the diagram of mineral abundance versus progress variable may be transformed into one relating mineral abundance and depth. Note that if diffusion or dispersion is important, the mass transfer approach has to be modified in a major way.

These equilibrium reaction path calculations can be extended to include a treatment of kinetics. As an example, calculations were carried out using the following simple rate laws:

$$
R_{i}=k_{i} \frac{\Delta G_{i}}{R T}
$$

where $\Delta G_{i}$ refers to the net free energy change of reaction (1) for the feldspar and similar reactions for gibbsite, kaolinite, and muscovite and $k_{i}$ is a rate constant. The equilibrium calculation carried out before is equivalent to assuming that the $\mathrm{k}_{i} / \mathrm{k}_{\text {feldspar }}$ ratio for gibbsite, kaolinite and mica is huge. It is quite instructive to actually see the relation of the kinetic paths and their approach to the equilibrium model as the rate constants are increased. Therefore, a series of kinetic mass transfer calculations have been carried out and shown in figures 3 to 7 . In each case, the rate constants, $k_{i}$, for all the product mineral phases was taken as the same and the ratio of this rate constant to the feldspar reaction rate constant was varied from a high value of 1000 times greater to a 
SURFACE

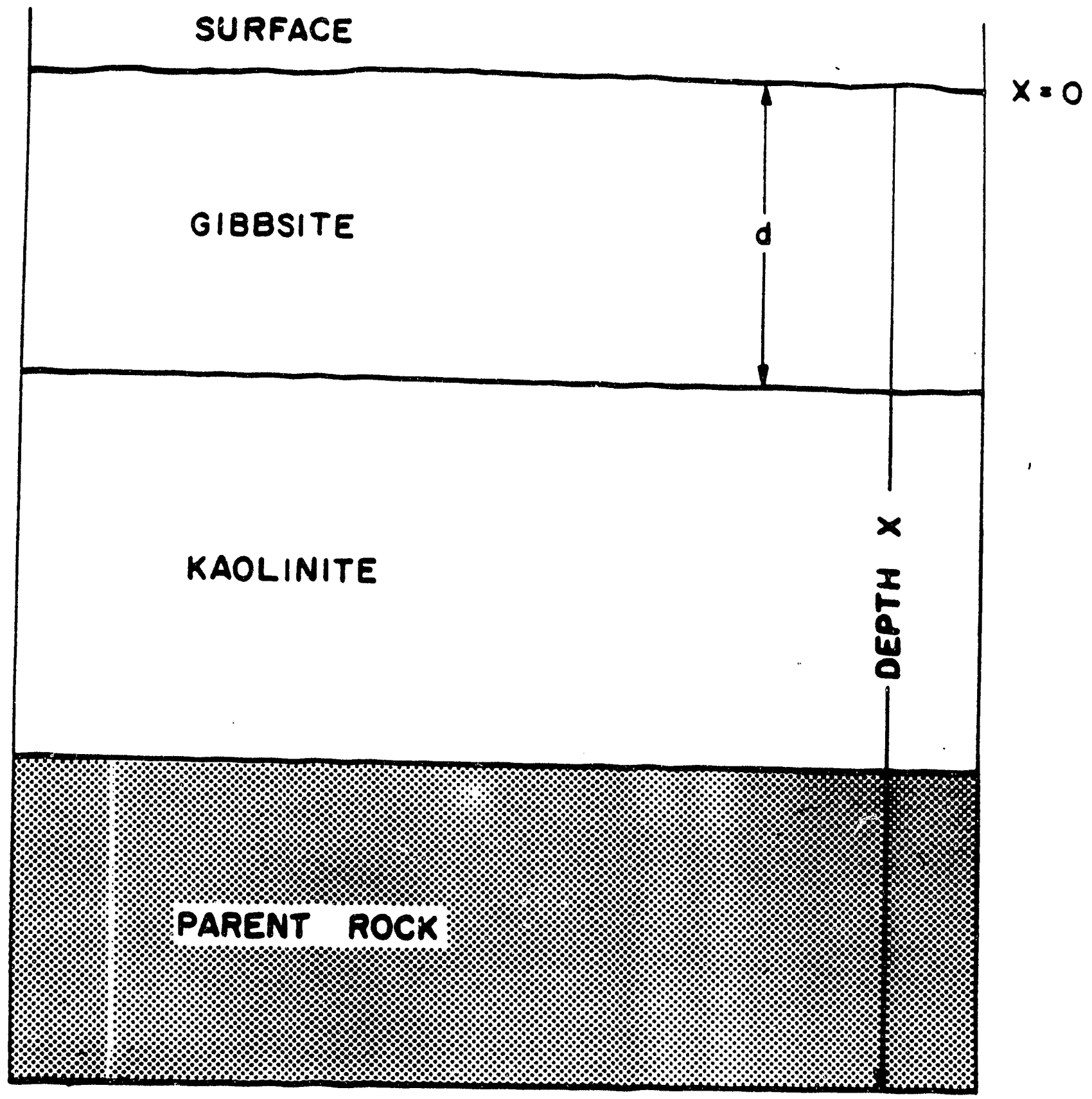

Figure 2 

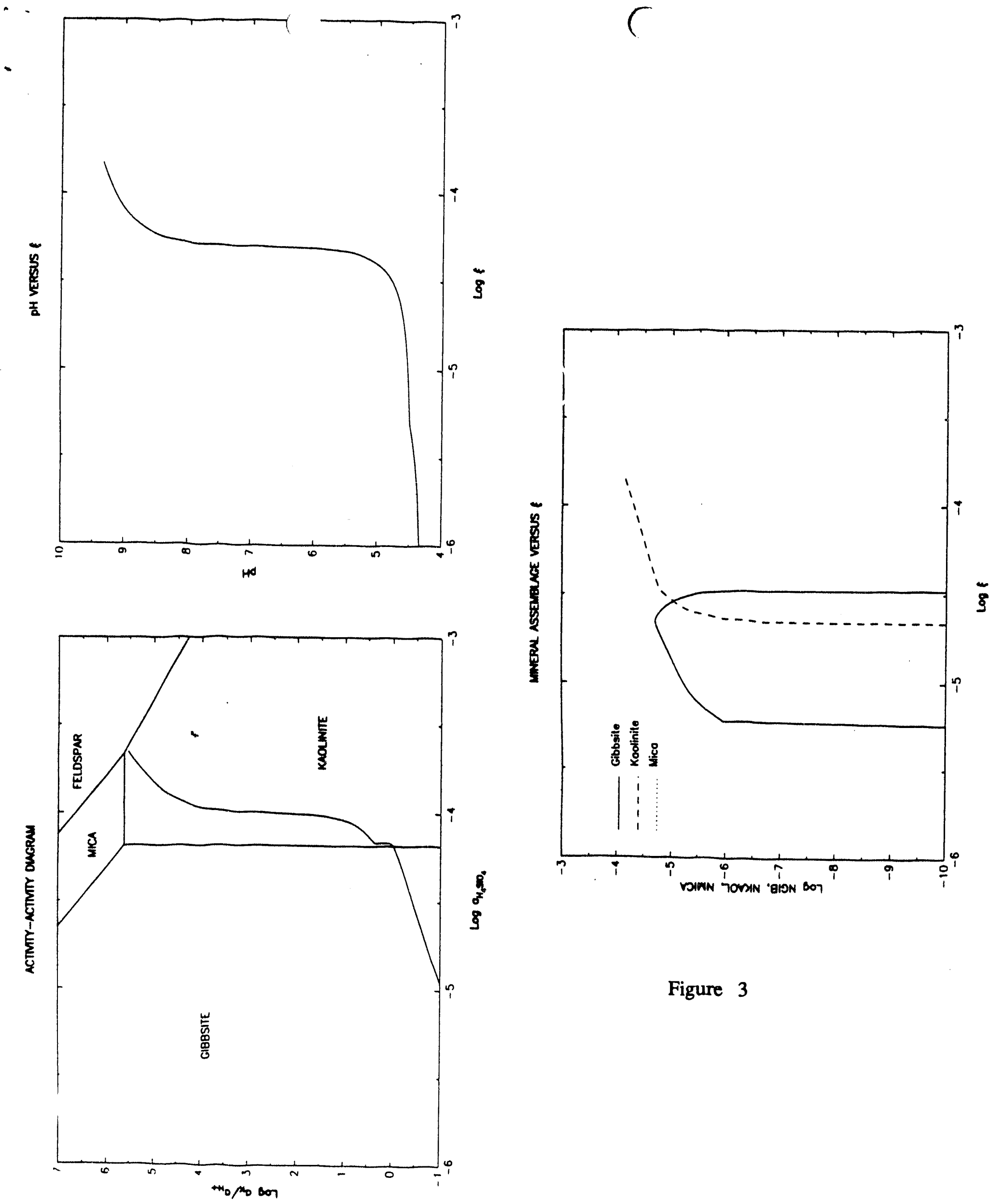

Figure 3 


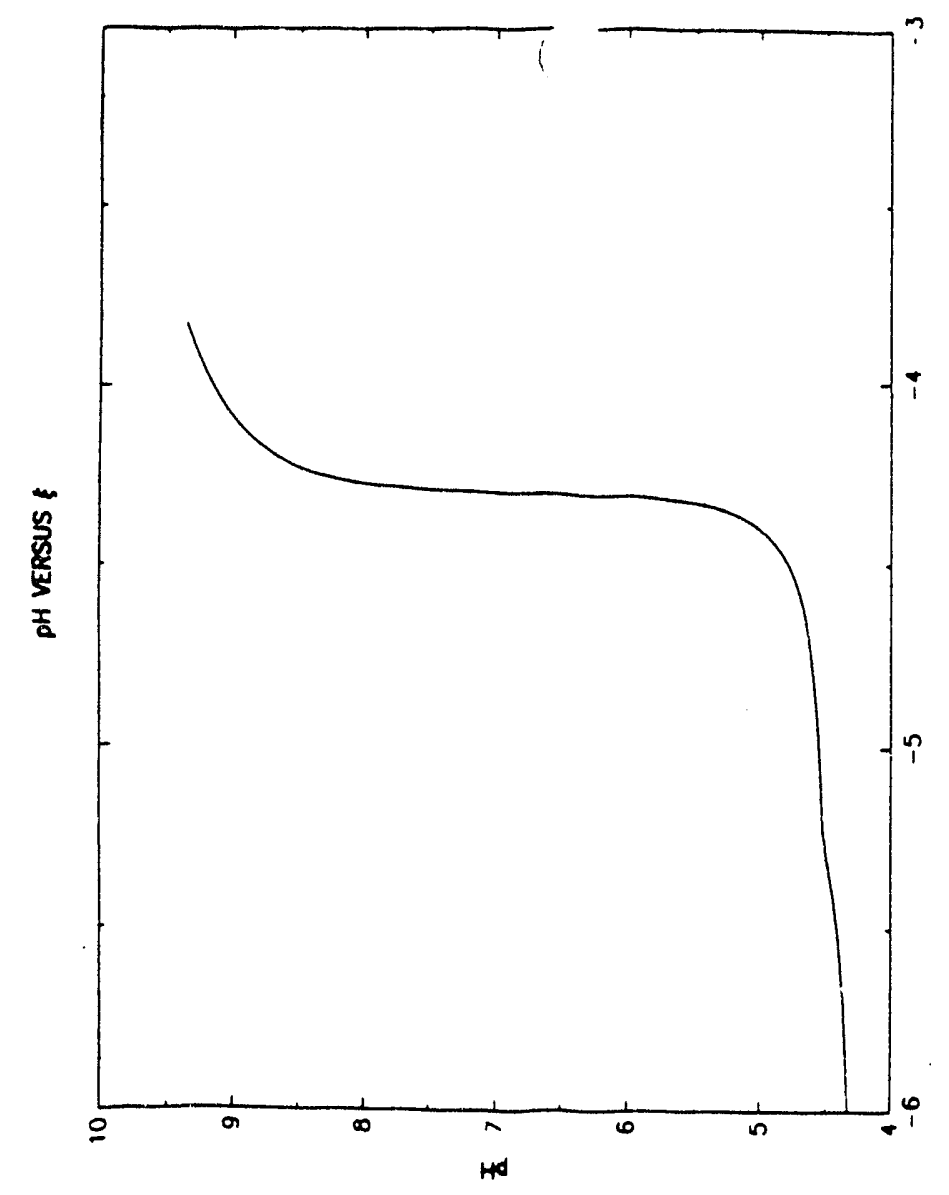

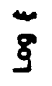

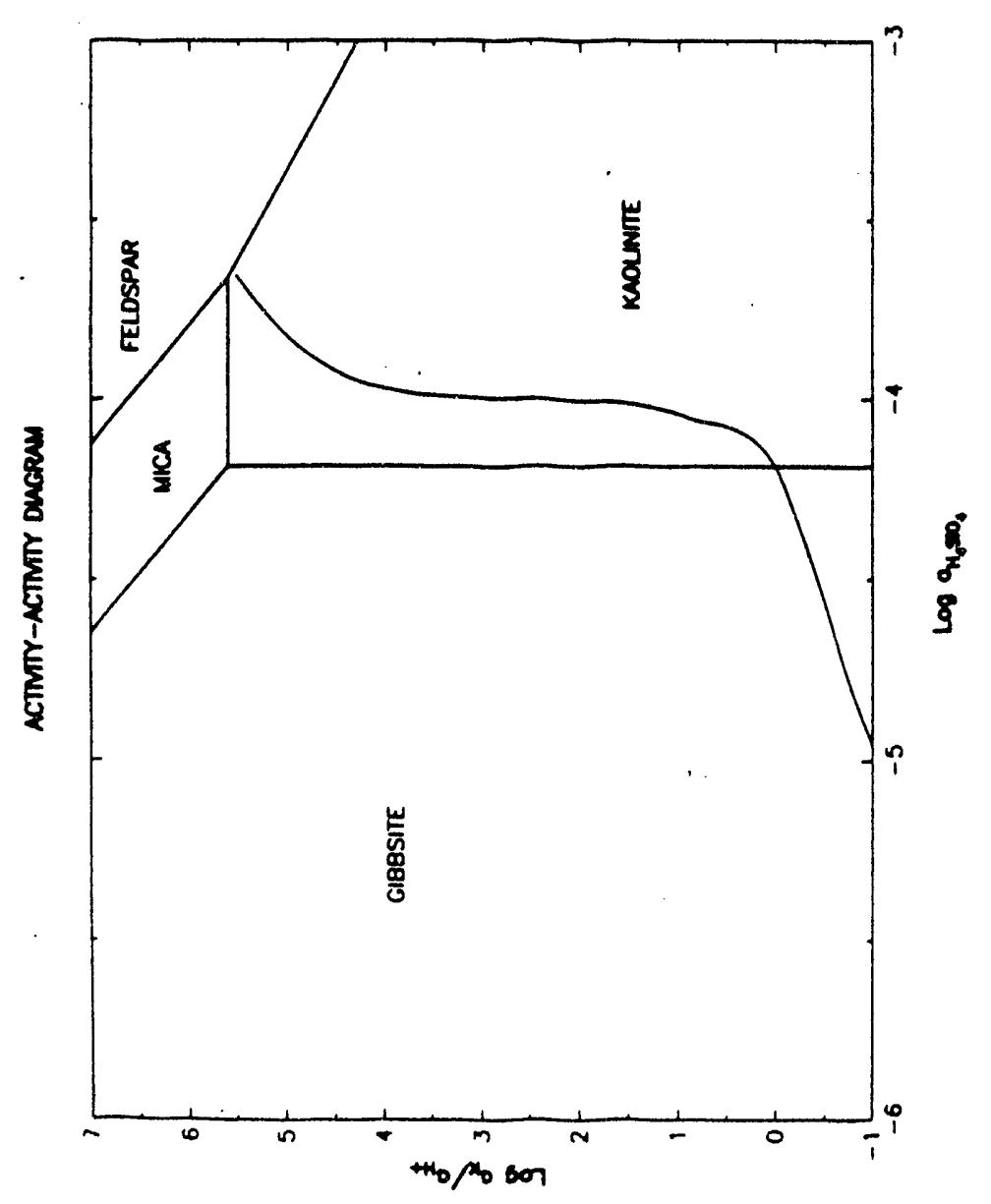

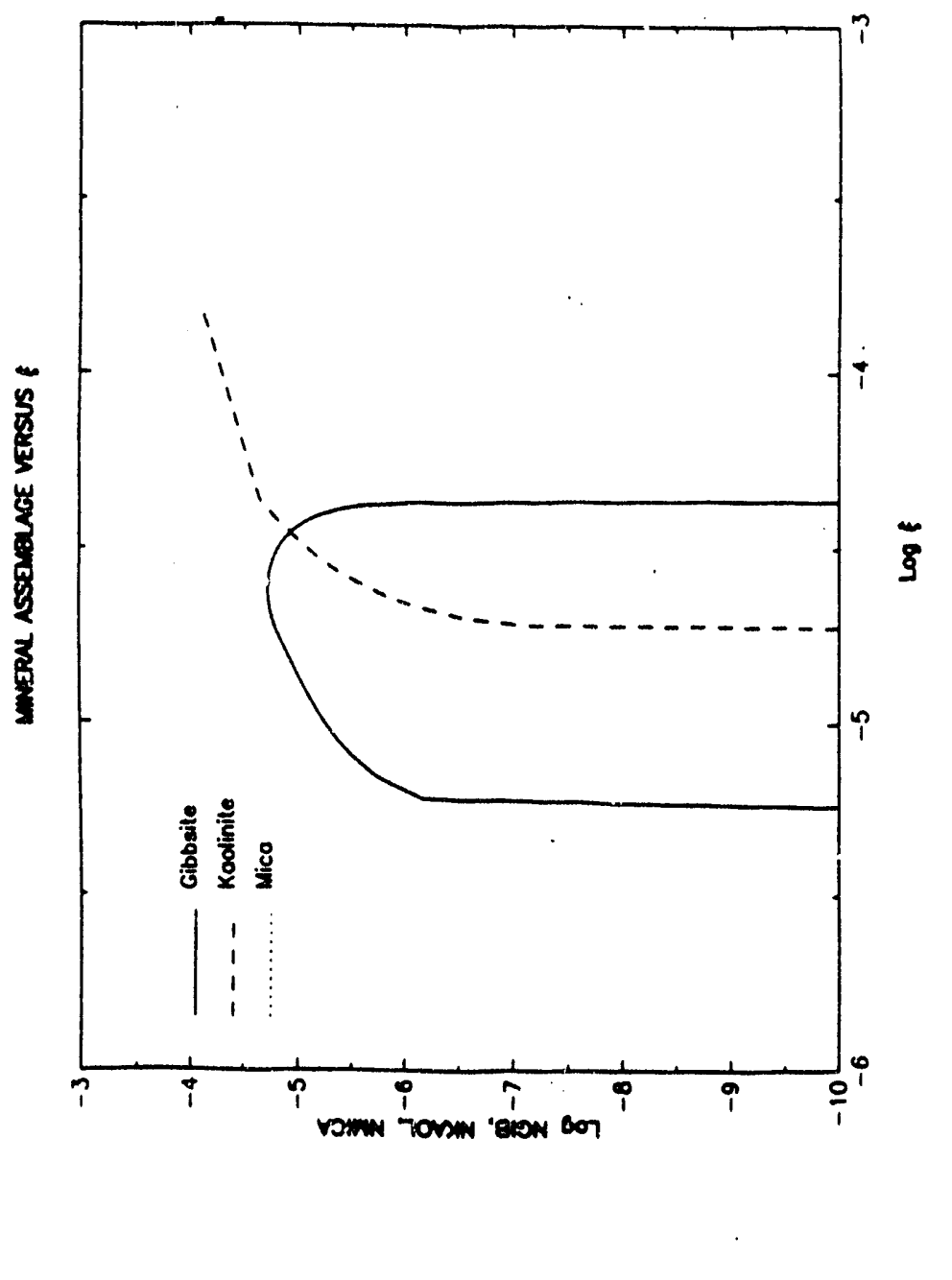

Figure 4 

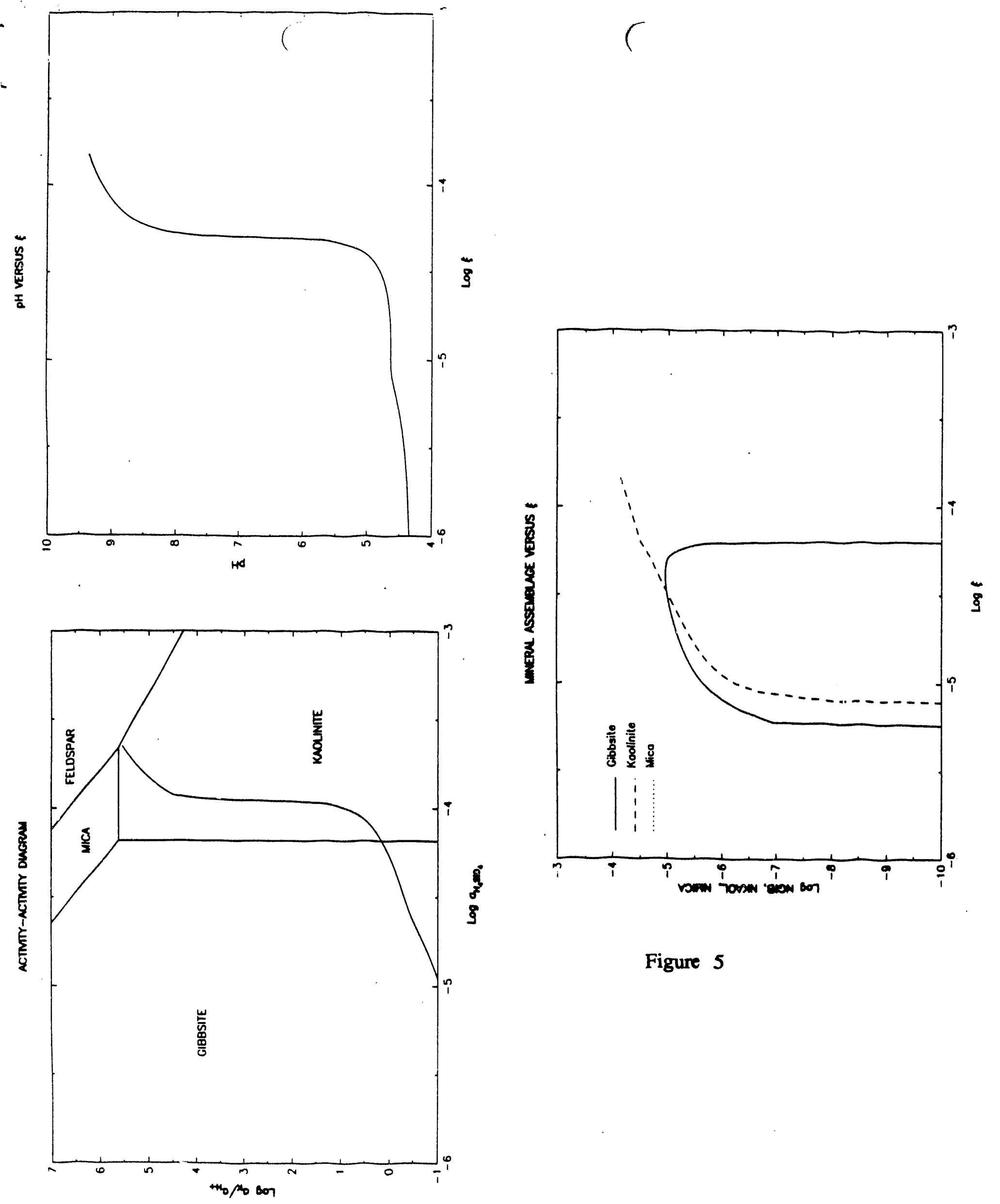

Figure 5 

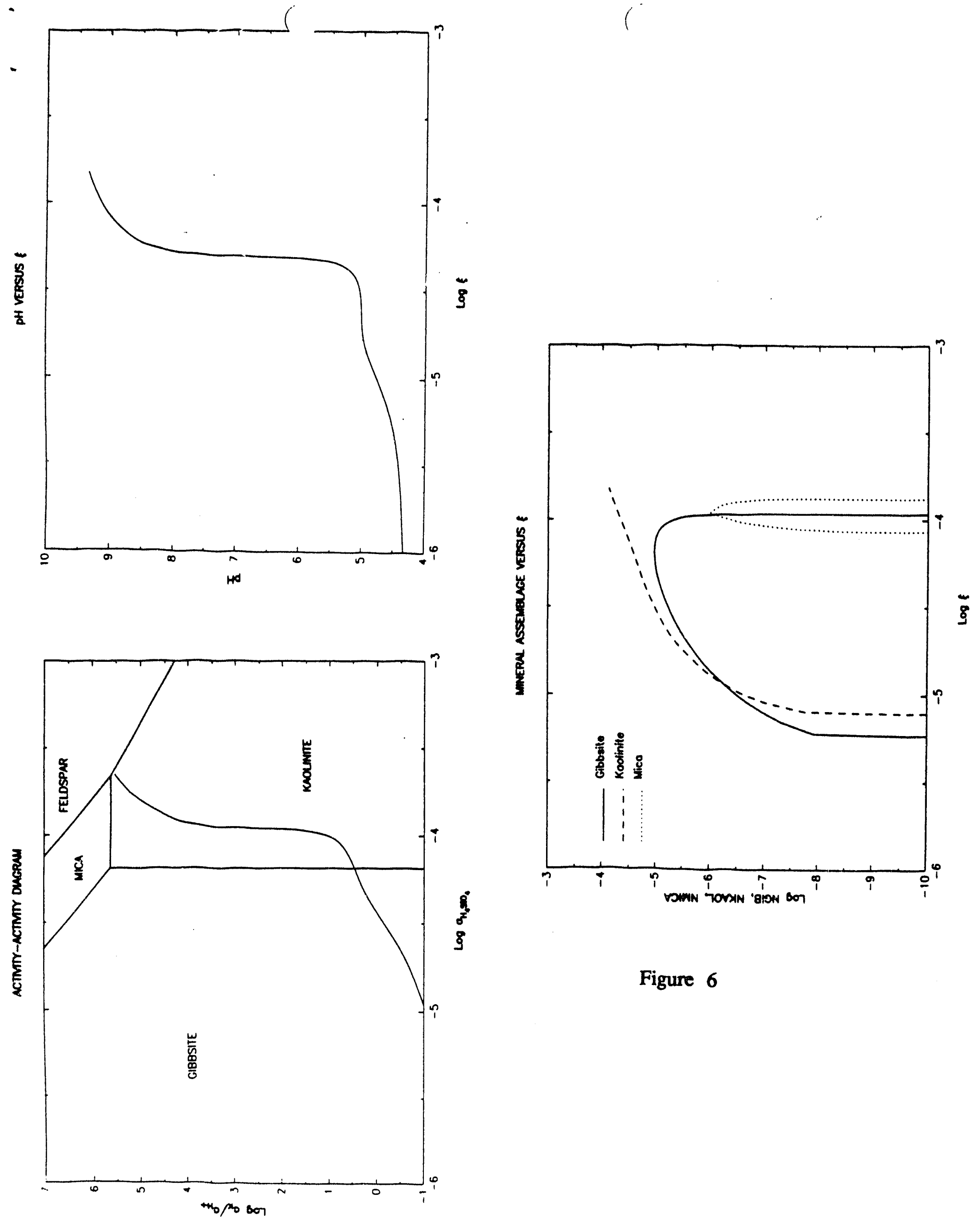

Figure 6 

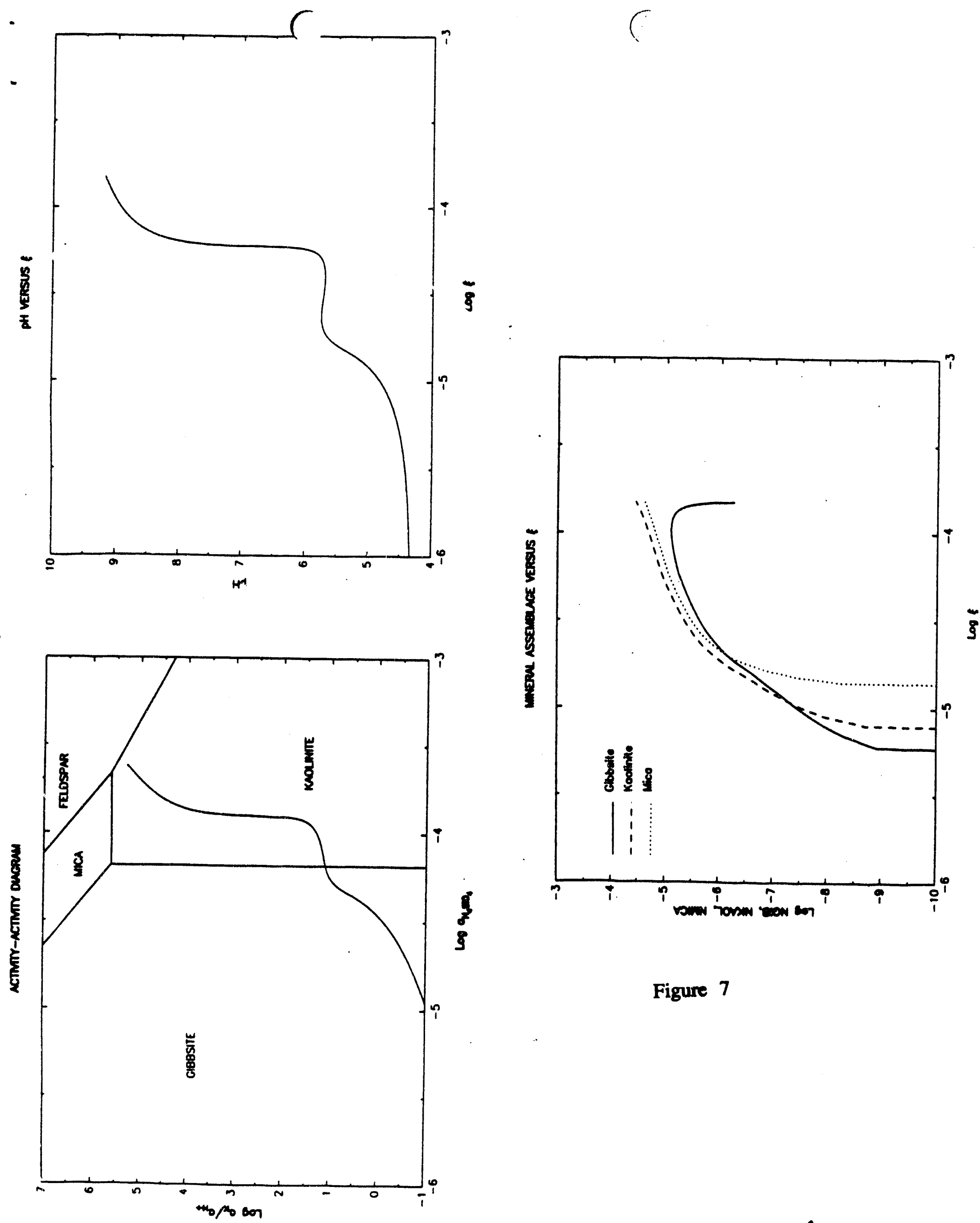

Figure 7 
low value of 0.1 . The results for $k_{i} / k_{\text {feld }}=1000$ are shown in figure 3 . In this case, the full kinetic treatment leads to a mass transfer calculation that is quite similar to the equilibrium case (i.e. only feldspar dissolution is kinetically sluggish). Howeve:, even for the case that $k_{i} / k_{f e l d}=100$, the kinetic treatment shows deviations from the classical equilibrium results (figure 4). In particular, the solution composition does not follow the boundaries of mineral pairs (e.g. gibbsite-kaolinite) and the region containing both gibbsite and kaolinite increases over that in the equilibrium case. Note that the changes in the mineralogy exhibit the strongest effects of the slow kinetics. At the slowest rates, Fig. 6 and 7, all three alteration minerals exhibit a variation that is quite different from the equilibrium one.

The recent experimental data obtained in our laboratories have shown that the relative kinetics for gibbsite/albite and kaolinite/albite rates are in the ratio 100.0 and 0.1 respectively (Nagy and Lasaga, 1982; Nagy, Blum and Lasaga, 1991; Burch, Nagy and Lasaga, 1992). Hence, the inclusion of kinetics is essential to understanding this important system. Obviously, the kinetic mass transfer calculations shown here must be extended into a general coupled flow and reaction model. This extension is part of the general program that has been developed at Yale and discussed in the next section.

\section{Coupled Flow-Reaction Model}

The approach to modeling reactive flow adopted in this project is to use numerical methods based on the finite difference method (Miller and Benson, 1983; Lichtner, 1985; Kirkner and Reeves, 1988; Reeves and Kirkner, 1988; Ague and Brimhall, 1989; Liu and Narasimhan, 1989a and 1989b; Steefel and Van Cappellen, 1990; Yeh and Tripathi, 1991; Steefel, 1992; Steefel and Lasaga, 1992). The common feature of these methods is that they break the domain of interest up into a series of discrete blocks or elements. A numerical solution is obtained instead by solving $N$ algebraic equation, where $N$ is the number of blocks or elements we have subdivided the problem into. The accuracy with which we can solve the governing set of partial differential equations depends in this case on the number of blocks or elements we use. In a multi-component chemical system, we also have to consider $M$ independent components, so that in practice the minimum number of equations to be solved at every time step is $N \times M$.

Figure 8 is a schematic representation of a finite difference formulation for a two dimensional system in which both hydrodynamic flow and chemical reactions occur. In this simple formulation, each grid point is directly linked to four nearest neighbors in the algebraic equation which describes the conservation of the field variable (e.g., enthalpy, fluid mass, or solute mass). Since the same configuration is used for every grid point within the domain (except at the boundary, where the geometry is slightly different), each grid point becomes indirectly linked to all the others when the set of algebraic equations are solved simultaneously. Within each volume, chemical reactions between the various aqueous species and between the species and the minerals in the rock take place. If the volume were closed, one could think of it conceptually, to use an experimental analogue, as a well-stirred batch reactor. Computationally, the equivalent would be a computer program like EQ3/EQ6 (Wolery. 1979) run as a closed system. Since each volume is open, however, fluxes from adjacent points influence the rates of reaction within the volume.

If, in addition, the hydrodynamics and heat transfer within the system can be determined using a similar discretization, then they can be coupled to the chemical reactions. This makes it possible to calculate realistic fluxes between the various subregions within 


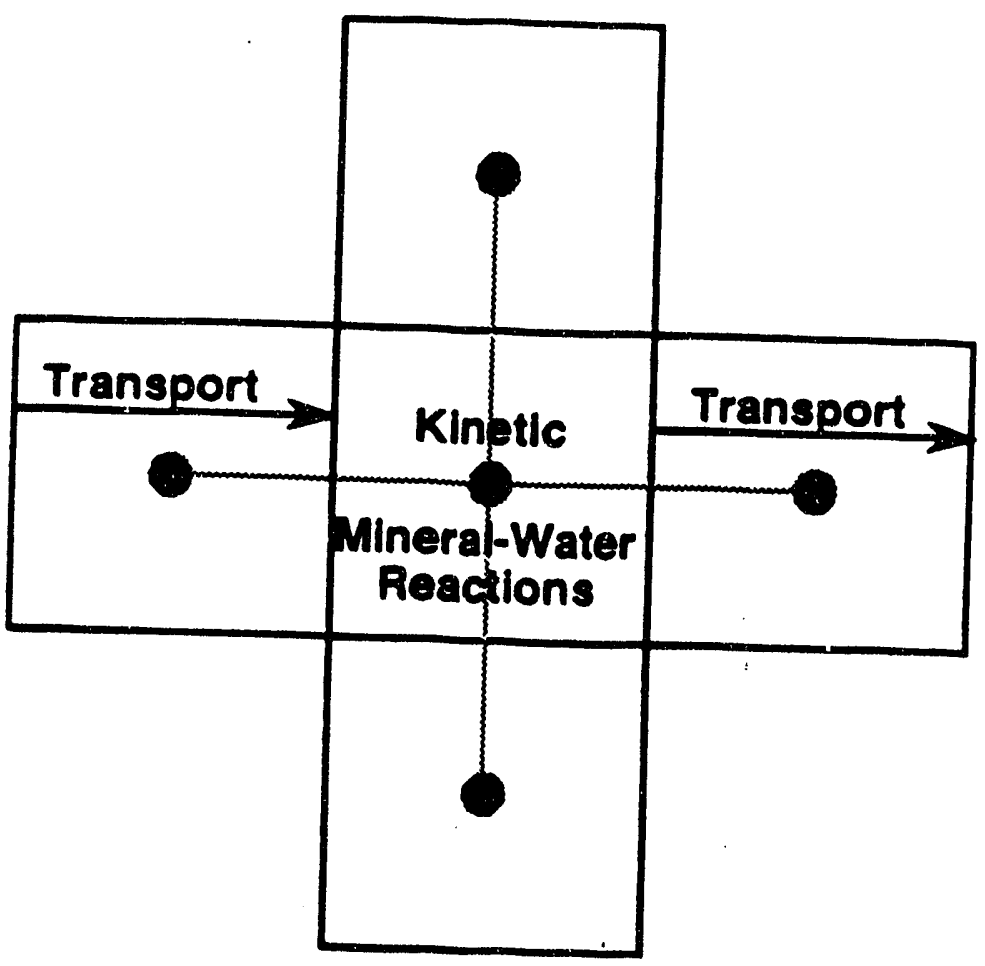

Figure 8 Schematic representation of the finite difference approach to modeling coupled reaction and transport in two dimensions. At each grid point within the discretized system, a set of differential equations conserving the masses of the chemical components in the system is solved, taking into account both transport (advection, dispersion, and diffusion) and mineral-water and complexation reactions. Each grid point is linked to four nearest neighbors in the case where the longitudinal and transverse dispersivities are the same. Thus, the reactions at any point in space are influenced or even driven by the fluxes from neighboring points. 
the domain of interest. We can see that by linking all of these blocks together in the finite difference approach, we have a description of the dynamics of the entire system (even if approximate because of the discretization). Obviously, however, we must prescribe a boundary to the system at some point where either the flux or the concentration of the chemical components in the system are fixed (otherwise the problem is unsolvable). The point of enlarging our system to include a whole series of contiguous blocks is to be able to describe the flux into and out of some region in the middle of our system (e.g., the high temperature portion of a hydrothermal system) only in terms of the dynamics of the system and in terms of boundary conditions that presumably we are more sure about (for example, the chemical composition of rainwater seeping into the system from above). In this regard, the motivation for solving the full partial differential equation instead of the simpler, batch reaction path equations in which mixing is included, is that, in general, both the rate of mixing (which is determined by the magnitude of the dispersive flux) and the composition of the mixed fluid (which is influenced by reactions in neighboring blocks) are a priori unknown.

\subsection{Mathematical Formulation}

A partial differential equation describing the conservation of solute mass for a system which inclu des both transport and reaction in a porous medium can be written

$$
\frac{\partial\left(\phi C_{\imath}\right)}{\partial t}+\operatorname{div}\left(\mathbf{J}_{\text {disp }}+\mathbf{J}_{a d v}\right)=R_{\mathrm{t}} \quad\left(\imath=1,2, \ldots N_{\text {tot }}\right)
$$

where $C_{z}$ is the concentration of some species in solution (in units of moles per unit volume solution), $N_{\text {tot }}$ is the total number of aqueous species, $\mathbf{J}_{\text {disp }}$ is the combined dispersivediffusive flux (in units of moles per unit area rock per unit time), $\mathbf{J}_{a d v}$ is the advective flux, and $R_{t}$ (in units of moles per unit volume rock per unit time) is the total reaction rate of species 2 in solution. Note that the porosity, $\phi$, is imbedded in both the flux terms and the reaction terms as described below. If the system is not isothermal, then the concentration (in moles per unit volume fluid) can be written in terms of the fluid density, $\rho_{f}$, and the molality, $m_{z}$,

$$
C_{\mathbf{\imath}}=\rho_{f} m_{\mathrm{i}}
$$

The reaction term $R_{1}$ can be divided into dissolution-precipitation (heterogeneous) reactions, $R_{z}^{\text {min }}$, and aqueous (homogener,us) reactions, $R_{z}^{a q}$, such that

$$
R_{\mathrm{z}}=R_{\mathrm{t}}^{\mathrm{min}}+R_{\mathrm{t}}^{a q}
$$

if sorption reactions are neglected. The reaction rate is written here in terms of a unit volume of rock but could be written equivalently per unit volume of fluid if it were then multiplied by the porosity, $\phi$. The advective flux is given by

$$
\mathbf{J}_{a d v}=\mathbf{u} C_{\imath},
$$

where $\mathbf{u}$ is the Darcian flux. The Darcian flux is related to the true velocity of the fluid, $\mathbf{v}$, by

$$
\mathbf{u}=\phi \mathbf{v}
$$


The dispersive flux is given by (Marsily, 1986)

$$
\mathbf{J}_{\text {disp }}=-\mathbf{D} \bullet \operatorname{grad} C_{\imath},
$$

where $\mathbf{D}$ is the dispersion tensor defined as the sum of the mechanical or kinematic dispersion $\mathbf{D}_{\mathbf{h}}$ and of the product of the porosity, $\phi$, and the molecular diffusion coefficient, $\mathbf{D}_{\mathbf{d}}^{*}$ (Marsily, 1986)

$$
\mathbf{D}=\mathbf{D}_{\mathbf{h}}+\phi \mathbf{D}_{\mathbf{d}}^{*}
$$

The kinematic dispersion is usually written as

$$
\mathbf{D}_{\mathbf{h}}=\mathbf{u} \alpha=\phi \mathbf{v} \alpha,
$$

where $\alpha$ is the dispersivity of the porous medium (Bear, 1979; Marsily, 1986).

The heterogeneous reaction term $R_{z}^{\text {min }}$ can be written more explicitly as the sum of all the individual mineral-water reactions which affect the concentration of the 2 th species

$$
R_{i}^{\text {min }}=-\sum_{k=1}^{N_{m}} \nu_{s k} r_{m},
$$

where $r_{m}$ is the rate of precipitation or dissolution of mineral $k$ per unit volume rock, $\nu_{\imath} k$ is the number of moles of $\imath$ in mineral $k$, and $N_{m}$ is the number of minerals present in the rock. By convention, $r_{m}$ is generally taken as positive for precipitation and negative for dissolution.

Equation 4 provides a general formulation for the conservation of solute mass which makes no assumptions of chemical equilibrium. If it can be assumed that the varions aqueous species are in chemical equilibrium, however, it is possible to reduce the number of independent concentrations, that is, the number that actually need to be solved for. Mathematically, this means that in a system containing $N_{\text {tot }}$ aqueous species, the number of independent chemical components in the system $N_{c}$ is reduced from the total number of species by the $N_{x}$ linearly independent chemical reactions between them (for further discussion, see Hooyman, 1961; Aris, 1965; Bowen, 1968; Van Zeggeren and Storey, 1970; Reed, 1982; 'Lichtner, 1985; Kirkner and Reeves, 1988). This leads to a natural partitioning of the system into $N_{c}$ primary or basis species, designated here as $C_{\jmath}$, and the $N_{x}$ secondary species, referred to as $X_{\imath}$ (Reed, 1982; Lichtner, 1985; Kirkner and Reeves, 1988). The reversible chemical reactions between the primary and secondary species take the form

$$
A_{\imath} \rightleftharpoons \sum_{j=1}^{N_{c}} \nu_{\imath \jmath} A_{\jmath} \quad\left(\imath=1, \ldots, N_{x}\right),
$$

where the $A$, and the $A_{\mathfrak{t}}$ are the chemical formulas of the primary and secondary species respectively and $\nu_{i j}$ is the number of moles of primary species $\}$ in one mole of secondary species $\imath$. It should be noted here that the partitioning between the primary and secondary species is not unique, that is, we can write the chemical reactions in more than one way. The reversible reactions provide an algebraic link between the primary and secondary species via the law of mass action for each reaction 


$$
X_{\imath}=K_{\imath}^{-1} \gamma_{\imath}^{-1} \prod_{\jmath=1}^{N_{c}}\left(\gamma_{\jmath} C_{\jmath}\right)^{\nu_{\imath},} \quad\left(\imath=1, \ldots, N_{x}\right)
$$

where the $K_{2}$ are the equilibrium constants of the reaction given in Equation 13, written here as the destruction of one mole of the secondary spe.ies. Given this partition between primary and secondary species, the governing partial differential equations can be written as

$$
\begin{aligned}
& \frac{\partial\left(\phi C_{\jmath}\right)}{\partial t}+\operatorname{div}\left(\mathrm{u} C_{\jmath}-\mathrm{D} \bullet \operatorname{grad} C_{\jmath}\right)=R_{\jmath}^{\min }+R_{\jmath}^{a q} \quad\left(\jmath=1, \ldots, N_{c}\right) \\
& \frac{\partial\left(\phi X_{\imath}\right)}{\partial t}+\operatorname{div}\left(\mathbf{u} X_{\imath}-\mathbf{D} \bullet \operatorname{grad} X_{\imath}\right)=R_{\imath}^{m i n}+R_{\imath}^{a q} \quad\left(\imath=1, \ldots, N_{x}\right) .
\end{aligned}
$$

Equation 13, however, implies that the rate of production of a primary component $\jmath$ due to homogeneous reactions can be written in terms of the sum of the total rates of production of the secondary species (Kirkner and Reeves, 1988)

$$
R_{\jmath}^{a q}=-\sum_{i=1}^{N_{x}} \nu_{\imath \jmath} R_{\imath}
$$

Equation 17 suggests that one can think of a mineral dissolving, for example, as producing only primary species which then equilibrate instantly with the secondary species in the system. Using Equation 17, the rates of the reversible reactions ca: be eliminated. Multiplying Equation 16 by $\nu_{i}$, and summing over all of the $N_{x}$ secondary species yields

$$
\frac{\partial}{\partial t}\left[\phi\left(\sum_{\imath=1}^{N_{x}} \nu_{\imath} X_{i}\right)\right]+\operatorname{div}\left[\mathrm{u}\left(\sum_{i=1}^{N_{x}} \nu_{\imath}, X_{\imath}\right)-\mathrm{D} \bullet \operatorname{grad}\left(\sum_{\imath=1}^{N_{x}} \nu_{\imath}, X_{\imath}\right)\right]=-R_{j}^{a q}
$$

Adding Equation 15 and Equation 18 gives a set of partial differential equations with $N_{c}$ unknowns

$$
\begin{gathered}
\frac{\partial}{\partial t}\left[\phi\left(C_{\jmath}+\sum_{i=1}^{N_{x}} \nu_{\imath} X_{\imath}\right)\right]+ \\
\operatorname{div}\left[\mathbf{u}\left(C_{\jmath}+\sum_{\imath=1}^{N_{x}} \nu_{\imath} X_{\imath}\right)-\mathbf{D} \bullet \operatorname{grad}\left(C_{\jmath}+\sum_{i=1}^{N_{x}} \nu_{\imath \jmath} X_{\imath}\right)\right]=R_{\jmath}^{\min } \quad\left(\jmath=1, \ldots, N_{c}\right)
\end{gathered}
$$

In this particular example, only the term $R_{j}^{\min }$ remains on the right hand side of Equation 19 because we have assumed that they are the only irreversible reactions. In the event that any of the homogeneous reactions are irreversible as well, they can be included on the right hand side in the same fashion (Lichtner, 1985).

If a total concentration, $U_{\jmath}$, is defined (Reed, 1982; Lichtner, 1985; Kirkner and Reeves, 1988) 


$$
U_{3}=C_{3}+\sum_{i=1}^{N_{x}} \nu_{\imath} X_{\imath}
$$

then the governing differential equations can be written in terms of the total concentrations (Kirkner and Reeves, 1988)

$$
\frac{\partial\left(\phi U_{\jmath}\right)}{\partial t}+\operatorname{div}\left(\mathbf{u} U_{\jmath}-\mathbf{D} \bullet \operatorname{grad} U_{\jmath}\right)=R_{\jmath}^{\min } \quad\left(\jmath=1, \ldots, N_{c}\right) .
$$

As pointed out by Reed (1982) and Lichtner (1985), the total concentrations can usually be interpreted in a straightforward fashion as the total elemental concentrations (e.g., total aluminum in solution), but in the case of $\mathrm{H}^{+}$and redox species, the total concentration has no simple physical meaning and the total concentrations may take on negative values.

\subsection{Mineral Precipitation/Dissolution Rate Laws}

In a general form, the rate of growth or dissolution of a mineral in aqueous solution can be expressed as

$$
\text { rate }=A k f\left(a_{\imath}\right) f(\Delta G)
$$

where $A$ is the reactive surface area of the mineral of interest (e.g., $\mathrm{m}^{2}$ per $\mathrm{m}^{3}$ total rock), $k$ is the rate constant (moles formula units mineral per $\mathrm{m}^{2}$ per second), $f\left(a_{\imath}\right)$ is some function of the activities of the individual ions in solution, and $f(\Delta G)$ is some function of the free energy of the solution. The functions, $f\left(a_{z}\right)$, represent the inhibiting or catalyzing effect of various ions in solution which should be considered separately from the effect of the saturation state per se. The experimental part of our project has been determining the kinetic details related to equation (22). Our early models have used the following form for the rate law of crystal growth and dissolution of a mineral (see Lasaga, 1981; Aagaard and Helgeson, 1982; Lasaga, 1984; Steefel and Van Cappellen, 1990)

$$
r_{m}=A_{m} k_{m}\left(\prod_{i=1}^{N_{c}+N_{x}} a_{i}^{p}\right)\left[\left(\frac{Q_{m}}{K_{m}}\right)^{\mathcal{M}}-1\right]^{n}
$$

where $k$ is either the growth or dissolution rate constant, $a_{\mathfrak{t}}$ is the activity of an inhibiting or catalyzing species raised to an empirically determined power $p$, and $\mathcal{M}$ and $n$ are two positive numbers which are also normally determined by experiment. In this expression $Q_{m}$ is the ion activity product written as

$$
Q_{m}=\prod_{\jmath=1}^{N_{c}} a_{j}^{\nu_{m}}
$$


and $K_{m}$ is the equilibrium constant for the mineral-water reaction written as the destruction cf one mole of mineral $m$. The appropriate functional form for the dependenre of the rate on the saturation state of the solution is the subject of a number of ongoing studies at our lab (e.g., Nagy and others, 1990; Van Cappellen and Berner, 1991; Nagy and others, 1991; Nagy and Lasaga, 1992, Burch and others, 1993). Nonlinear rate laws for the mineral-water reactions (i.e., $m$ and/or $n \neq 1$ ) can have a profound effect on the behavior of a reactive flow system, particularly where they result in a significant decrease in the reaction rates close to equilibrium (Steefel and Van Cappellen, 1990; Kerrick and others, 1991). Steefel and Van Cappellen (1990) used nonlinear rate laws for some of the minerals in their simulation of kinetically controlled weathering and found that they appeared to give more reasonable crystal growth rates than did the linear forms. A rigorous analysis of the effects of nonlinear rate laws on the behavior of reactive flow systems is a subject for future investigations. In our initial simulations, a linear form for all of the reactions are used (that is, both $m$ and $n=1$ ).

The temperature dependence of the reaction rate constant can be expressed reasonably well via an Arrhenius equation (Lasaga, 1984). Since many rate constants are reported at $25^{\circ} \mathrm{C}$, it may be more convenient to write the rate constant at some temperature as

$$
k=k_{25} \exp \left[\frac{-E_{a}}{R}\left(\frac{1}{T}-\frac{1}{298.15}\right)\right]
$$

where $E_{a}$ is the activation energy, $k_{25}$ is the rate constant at $25^{\circ} \mathrm{C}, R$ is the gas constant, and $T$ is temperature in the Kelvin scale.

Of the parameters which enter into the rate expression for a heterogeneous reaction, the reactive surface area or rock texture is in general the least constrained in natural systems. This is primarily because the surface areas of the reacting minerals are not constant with time (Steefel and Van Cappellen, 1990) or because the permeability structure of the medium has an effect on how much rock surface area is encountered by a particular volume of fluid. A rock permeability which is dominated by a few fractures of relatively wide aperture, for example, has only a small amount of surface area compared to the volume of fluid passing through it. The permeability structure of the rock may account for much of the apparent discrepancies betwen laboratory and field-determined mineral dissolution rates. It is clear that the amount of reactive surface area in real geological systems will turn out to be a complex function of the properties of the particular rock of interest, and somewhat like hydrodynamic dispersion, will have to be determined through field studies on a case by case basis.

In contrast to primary minerals, surface area in the case of secondary minerals is initially created by nucleation and is subsequently modified by crystal growth and dissolution and by crystal ripening. Time-dependent nucleation, crystal growth and dissolution, and ripening results in a distribution of grain sizes which must be accounted for in order to accurately determine secondary mineral surface areas. Steefel and Van Cappellen (1990) demonstrated that nucleation of new crystals to create surface area is potentially the 
dominant kinetic effect in low temperature systems. They showed that because of the extremely rapid rate of surface area generation above the nucleation barrier, it was not possible to maintain such levels of supersaturation for other than very short periods of

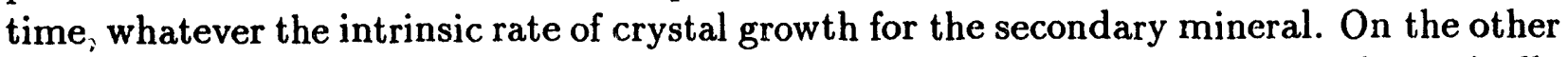
hand, at saturations below the nucleation barrier, the formation of thermodynamically stable secondary minerals is often extremely slow because the process must proceed via the formation of a precursor.

A simpler approach can be taken which does not require that a distribution of crystal sizes be computed. Nucleation is envisioned as a single event which produces between, for example, $10^{7}$ to $10^{11}$ crystals of $0.1 \mu \mathrm{m}$ which then grow (or dissolve) all at the same rate (ripening, therefore, is neglected). In future developments, it will be important to include the effects of nucleation and ripening on a more routine basis in reaction-transport simulations in order to obtain more realistic kinetic descriptions of water-rock interaction. For primary minerals, the initial mineral surface areas are determined assuming that all of the grains have the same spherical geometry. The grain radii and the initial volume percentages of the minerals, then, determine the total mineral surface area in the rock. The linear growth rate, given by

$$
\frac{d r_{c}}{d t}=k_{m} V_{m}\left[\frac{Q_{m}}{K_{m}}-1\right]
$$

where $r_{c}$ is the crystal radius and where $V_{m}$ is the molar volume of the mineral, is used to calculate the changes in crystal size as a function of time (Lasaga, 1984). From this information, a time-dependent total mineral surface area can then be calculated (knowing the number of grains per unit volume rock). The change in the volume fraction of an individual mineral can also be calculated from the grain size or more directly from

$$
\frac{d \phi_{m}}{d t}=A_{m} \frac{d r_{c}}{d t}
$$

From this expression, the porosity of the medium can immediately be obtained (if compaction and dilation are neglected), since

$$
\phi=1-\sum_{m=1}^{N_{m}} \phi_{m} .
$$

\subsection{Implicit vs. Explicit Schemes}

In terms of the numerical methods which could be used to solve multi-component reactiontransport problems, the most important distinction is between explicit (often referred to as uncoupled or multi-step) methods, where the value of some unknown is calculated from information from the previous time step, and implicit (or coupled) methods, which need information from the next time step and therefore require the solution of a set of simultaneous equations. From a computational point of view, the decoupling of the various terms 
within the reaction-transport equation can be pursued at a number of levels, but the most common approach is to decouple the transport and the reaction terms (Oran and Boris, 1987; Yeh and Tripathi, 1989; Mangold and Tsang, 1991). This method, which is often referred to as the two-step method, involves alternately solving for the multi-component reaction term and the transport term.

In our work, we have chosen to pursue the fully coupled methods. It is important to consider some of the potential advantages of the coupled or global implicit methods (Kee et al., 1985). Of these, the most important are:

1. The global convergence properties of the fully coupled method may be better than the multi-step methods. Using a Newton method to solve the full set of equations, for instance, we expect to achieve quadratic convergence in the vicinity of a root, while we can expect linear convergence at best from the decoupled methods.

2. It is usually possible to take larger timesteps with a fully implicit or coupled routine because of its superior numerical stability. This is particularly true where the governing differential equations are stiff, that is, when the time step needed to maintain numerical stability is much smaller than the time step needed for an accurate solution (Press and others, 1986). Stiffness arises in these kinds of differential equations primarily because of the different time scales represented by fast versus slow chemical reactions.

Given the choice of an implicit or coupled method to solve the coupled reactiontransport equation, there are still a number of possible approaches which can be used. Following the notation of Lichtner (1992), we can introduce the differential operator

$$
\mathcal{L}\left(U_{\jmath}\right)=\left[\frac{\partial}{\partial t} \phi+\nabla \bullet(\mathbf{u}-\text { Dgrad })\right] U_{\jmath},
$$

and write the governing differential equations in terms of the total component concentrations as

$$
\mathcal{L}\left(U_{\jmath}\right)=R_{\jmath}^{\min } \quad\left(\jmath=1, \ldots, N_{c}\right),
$$

where the reaction term $R_{j}^{\text {min }}$ includes only irreversible (in our case, heterogeneous) reactions. Note that in this formulation, we assume that the diffusion coefficients are the same for all of the aqueous species (both primary and secondary). This makes the solution of the reaction-transport equation much simpler because the secondary species do not have to be individually transported.

As discussed by Kirkner and Reeves (1988), one way to proceed at this point would be to solve directly for the total soluble concentrations (the $U_{3}$ 's), thereby decoupling the solution of the differential equations from the speciation calculations. In this formulation (formulations $\mathrm{B}$ and $\mathrm{C}$ of Kirkner and Reeves, 1988), every iteration to solve for $U_{3}$ 
is preceded by an equilibrium speciation calculation to determine the concentrations of both primary and secondary species. On the basis of a number of preliminary benchmark tests (not reported here), however, it appears that that the most robust solution method directly couples the speciation calculations to the differential equations. A similar conclusion was reached by Reeves and Kirkner (1988) who carried out a much more extensive set of benchmarks. We proceed by substituting Equation 14 into Equation 19 to obtain an expression for the total soluble concentration in terms of the primary species alone. Substituting this result into Equation $\mathbf{3 0}$ gives (essentially the formulation A of Kirkner and Reeves (1988))

$$
\mathcal{L}\left[C_{\jmath}+\sum_{i=1}^{N_{x}} \nu_{i \jmath} \gamma_{s}^{-1} K_{z}^{-1} \prod_{\jmath=1}^{N_{c}}\left(\gamma_{\jmath} C_{\jmath}\right)^{\nu_{l} \jmath}\right]+\left(\prod_{l=1}^{N_{c}+N_{x}} a_{l}^{p}\right) \sum_{m=1}^{N_{m}} A_{m} k_{m}\left[\left(\prod_{\jmath=1}^{N_{c}}\left(\gamma_{\jmath} C_{\jmath}\right)^{\nu_{m},} K_{m}^{-1}\right)^{\mathcal{M}}-1\right]^{n}=0
$$

where the $\gamma_{s}$ 's and $\gamma_{3}$ 's are the activity coefficients for the secondary and primary species respectively. If Equation 31 is discretized on a grid using $M$ nodal points (see below), for instance, then the total number of equations to be solved is $N_{c} \times M$, where $N_{c}$ is the number of primary species in the system.

Since Equation 31 is a nonlinear function of the primary species, $C_{\jmath}$, an iterative scheme is required to solve it. Restricting ourselves to one dimensional transport for the sake of clarity, the problem becomes one of finding the roots for $N_{c} \times M$ functions of the form

$$
f\left(C_{1}^{k}, \ldots, C_{N_{c}}^{k}\right)+f\left(C_{1}^{k-1}, \ldots, C_{N_{c}}^{k-1}\right)+f\left(C_{1}^{k+1}, \ldots, C_{N_{c}}^{k+1}\right)=0 \quad\left(\imath=1, \ldots, N_{c} \times M\right),
$$

where the superscript $k$ refers to the concentrations of the primary species at nodal point $k$, and the superscripts $k-1$ and $k+1$ refer to the concentrations of the primary species at adjacent grid points. In the scheme employed here, the function of the $N_{c}$ primary concentrations at the $k t h$ grid point includes contributions from both the mineral-fluid (heterogeneous) reactions and the the complexation (homogeneous) reactions. In contrast, the functions at the $k-1$ and $k+1$ grid points include contributions only from the complexation reactions (since the differential equations are written in the terms of the total concentrations, $U_{j}$ (e.g., Equation 31). Note that the nonlinearity in the problem comes only from the reaction terms which couple the $N_{c}$ species at any one grid point and not from the coupling between grid points which in a finite difference formulation is linear. In two dimensions, any grid point will be linked to at least 4 and potentially 8 neighboring point, depending on the discretization scheme which is employed. The resulting set of nonlinear equations is then solved using Newton's method. The method consists of using a Taylor series expansion to linearize the problem and then iterating until the root of the equation is found (Rheinboldt, 1974; Press and others, 1986; Ortega and Rheinboldt, 1970; Ortega, 1990). 


\section{Program Structure - 2DREACT}

The structure of the code used to solve the set of governing differential equations is suminarized in Figure 9. After specifying the initial and boundary conditions, the code begins stepping through cime. A check is made at this time to see whether basis switching is needed at any grid point in the system. Within each time step, a series of Newton iterations is required because of the nonlinearity of the equations. Each Newton iteration consists of calculating the function residuals (the $f\left(x_{1}\right)$ 's given by Equation (32)) and the partial derivatives of these functions (the Jacobian elements) with respect to the unknown concentrations and then assembling and soiving the linear set of $N_{c} \times M$ algebraic equations in order to obtain the corrections to the component concentrations. As discussed above, the functions include both the contributions from homogeneous and heterogeneous reactions and from advective, dispersive, and diffusive transport which are evaluated at each grid point within the system. This is repeated until convergence to some desired tolerance is achieved, i.e., when the $f\left(x_{\imath}\right)$ 's are all reduced to some desired tolerance. It is usually not necessary to update the matrix of partial derivatives every iteration since the Jacobian matrix does not have to be exact. Instead, the Jacobian is typically recalculated when the number of Newton iterations required to achieve convergence exceeds some specified tolerance (e.g., Kirkner and Reeves, 1988). This method (referred to as a modified Newton method) can be particularly efficient since it is the assembly of the Jacobian elements that take a large part of the CPU time (Kee and others, 1985). Once convergence is achieved, the mineral volume fractions and mineral surface areas are updated. If the reaction-transport calculations are combined with a calculation of the flow and temperature field, these would normally be updated as well at this time. If one chooses to make use of the quasi-stationary state approximation (Lichtner, 1988), then the mineral volume fractions and surface areas are updated only when a quasi-stationary state with respect to the fluid concentrations has been achieved, rather than after every time step. At the end of each time step, whether the code is run in either the quasi-stationary or transient mode, an algorithm which computes the second derivative of the solute concentrations with respect to time is used to determine whether the time step should be increased or decreased. This algorithm provides a way of minimizing the time truncation error (if that is an issue in a particular problem) and also of controlling the size of the time step so as to maintain numerical stability. As the solute concentration profile relaxes to some quasi-steady state, the time step gradually increases to some specified maximum value (for example, 5 years in the simulations to follow.) 


\section{Program Structure}

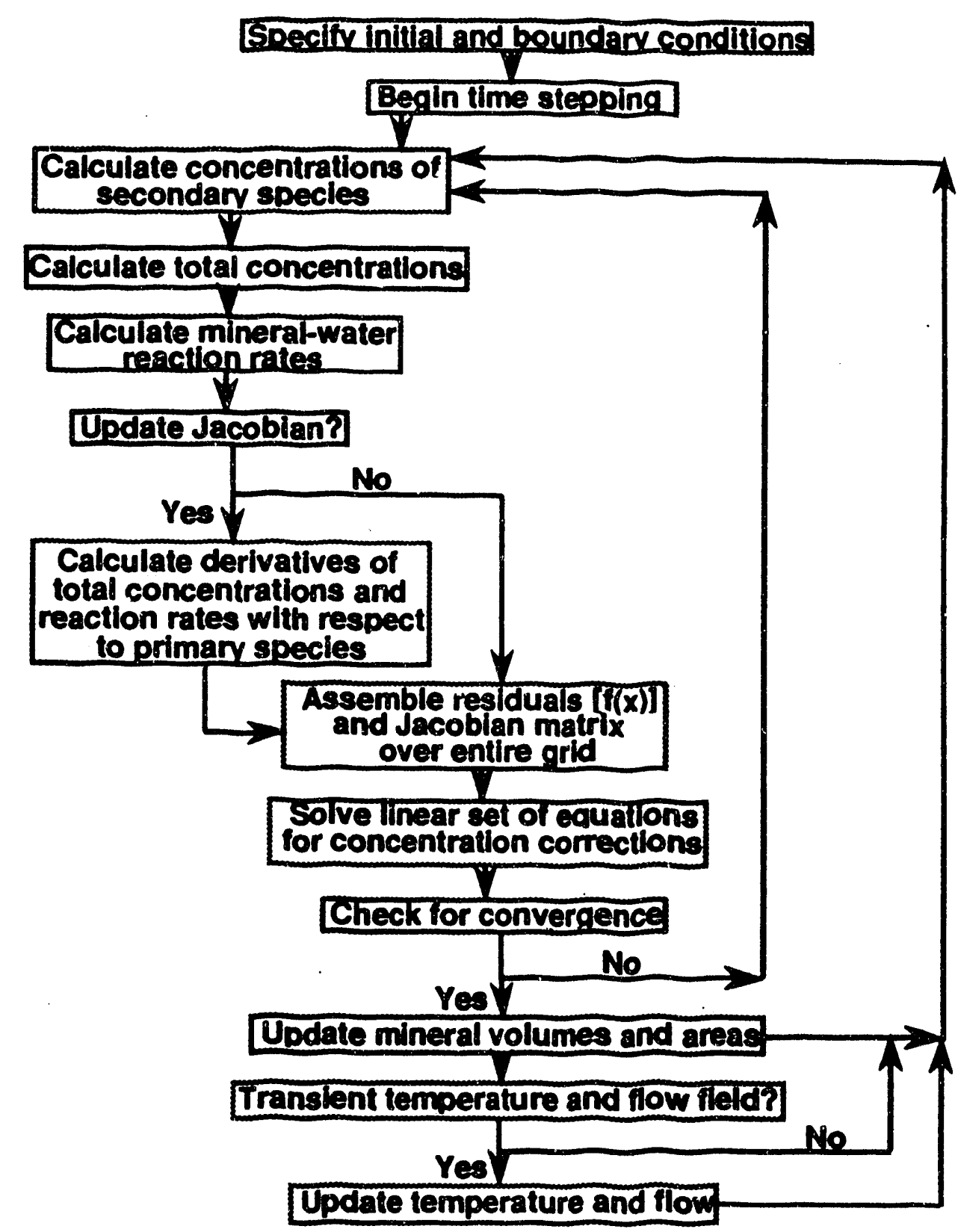

Figure 9: Program structure for the global implicit or one-step method used to solve the multi-component reaction-transport equation. Total concentrations and reaction rates are computed at each grid point within the domain. Although represented as a flow, many of the steps could be done in parallel. 


\section{Applications}

\subsection{Model for A Reacting Hydrothermal Convection Cell \\ 5.2 Boundary Conditions}

Since we are interested in a first order approach at this stage, we consider here what is probably the simplest possible model of a hydrothermal system, a Rayleigh-Benard convection cell. Figure 10 shows the boundary conditions which need to be specified for the problem in order to solve for the conservation of solute mass, the conservation of fluid mass, and conservation of energy. The sides of the system are symmetry boundaries which implies that there is no flux of any kind across them. The top and bottom boundaries have no mass or fluid flux across them and are fixed at constant temperatures of $50^{\circ} \mathrm{C}$ and $300^{\circ}$ respectively. Strictly speaking, one should include diffusive and dispersive mass transport across the lower and upper boundaries (no advective flux occurs across the boundary because the fluid flux is 0 ), but that is a relatively small correction given the vigor of the convection cells considered here.

The system described above can be visualized in terms of a region bounded by two horizontal heated plates. Such a system is usually characterized in terms of the nondimensional Rayleigh number (Turcotte and Schubert, 1982)

$$
R a=\frac{\alpha g \rho_{50}^{2} C_{p, f} k \dot{b} \Delta T}{\mu \lambda_{m}}
$$

where $\alpha$ is the thermal expansivity, $\rho_{50}$ is the reference density of water at $50^{\circ} \mathrm{C}, b$ is the vertical extent of the system, $\Delta T$ is the temperature between the upper and lower boundary. Other physical parameters used in the simulations and in the Rayleigh number calculations are given in Table 1.

\subsection{Initial Conditions}

We assume that the 2000 meter deep region of interest begins with a normal geothermal gradient of $25^{\circ} \mathrm{C} / \mathrm{km}$ and instantaneously acquires at $t=0$ its lower temperature of $300^{\circ} \mathrm{C}$. To complete the specification of the initial conditions, we need to give the initial total concentrations of the solutes or the mineral equilibria used to constrain the concentrations (Table 2) and the initial (or prinary) modal mineralogy of the rock. We restrict ourselves here to the relatively simple chemical system of $\mathrm{KCl}, \mathrm{NaCl}, \mathrm{Al}_{2} \mathrm{O}_{3}, \mathrm{SiO}_{2}$, and $\mathrm{H}_{2} \mathrm{O}$ (table 3 ). Since the initial fluid is not isothermal, the use of a mineral constraint results in differing initial concentrations which depend on the local temperature. The initial mineral volume percents vary slightly from one simulation to another so as to give differing initial porosities, but in each case the rock is initially made up of K-feldspar, albite, and quartz (i.e., a leucogranite). The full suite of minerals considered in the simulations includes K-feldspar, albite, quartz, muscovite, and kaolinite. 


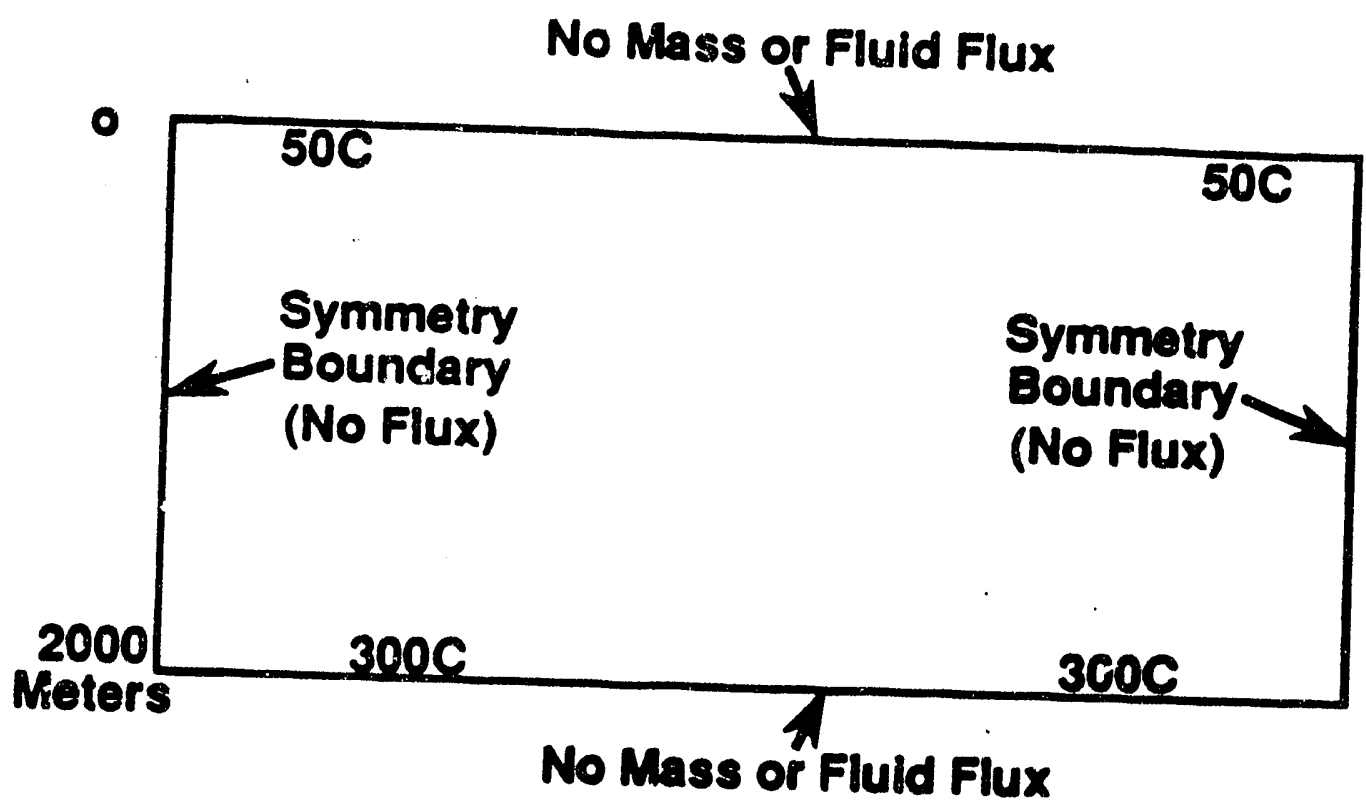

Figure 10 Boundary conditions used in simulations of a bydrotherinal convection cell 
It is important to point out that the choice of boundary and initial conditions can have a significant effect on the behavior of the system. The results which are given below, therefore, would be very different if, for instance, an influx of reactive fluid (e.g., rainwater or magmatic water) occurred across one of the boundaries.

Equilibrium constants for the heterogeneous and homogeneous reactions are calculated at each grid point using a fifth order polynomial fit to tabulated data. The log K's for the minerals are taken from Sverjensky and others (1990) who derived them from Berman (1988). The log K's for the complexation reactions are taken from the EQ3/EQ6 database (Wolery and others, 1990). Activity coefficients are calculated from an extended DebyeHuckel formulation (Wolery, 1983).

The rate data used in the calculations is given in Table 4 and are calculated from Equation 25. Equation 23 gives the form of the rate law used in all the calculations with both $\mathcal{M}$ and $n=1$ and with $p=0$ (i.e., "first-order" kinetics and no inhibitory or catalytic effects).

Table 1: Physical parameters used in hydrothermal simulations.

\begin{tabular}{ll}
\hline Top boundary temperature & $50^{\circ} \mathrm{C}$ \\
Lower boundary temperature & $300^{\circ} \mathrm{C}$ \\
Depth of system $(b)$ & $2000 \mathrm{~m}$ \\
Dirpersivity $\left(\alpha_{L}=\alpha_{T}\right)$ & $1 \mathrm{~m}$ \\
In itial mineral surface areas & $100 \mathrm{~m}^{-1}$ \\
T'uermal conductivity $\left(\lambda_{m}\right)$ & $3 \mathrm{~W} \mathrm{~m}-1{ }^{\circ} \mathrm{K}^{-1}$ \\
Heat capacity of fluid $\left(C_{p, f}\right)$ & $4200 \mathrm{~J} \mathrm{~kg}^{-1}{ }^{\circ} \mathrm{K}^{-1}$ \\
Heat capacity of rock $\left(C_{p, m}\right)$ & $1005 \mathrm{~J} \mathrm{~kg}^{-1}{ }^{\circ} \mathrm{K}^{-1}$ \\
Average rock density $\left(\rho_{m}\right)$ & $2600 \mathrm{~m}^{3} \mathrm{~kg}^{-1}$ \\
Reference fluid density $\left(\rho_{50}\right)$ & $1000 \mathrm{~m}^{3} \mathrm{~kg}^{-1}$ \\
Gravitational constant $(g)$ & $9.8 \mathrm{~m} \mathrm{~s}^{-2}$ \\
Thermal expansivity $(\alpha)$ & $10^{-3}{ }^{\circ} \mathrm{K}^{-1}$ \\
Average viscosity $(\mu) \dagger$ & $10^{-4} \mathrm{~Pa} \mathrm{~s}^{\dagger}$
\end{tabular}

† Value used in Rayleigh number calculations. A temperature-dependent viscosity is used in the numerical simulations. 
Table 2: Total solute concentrations and mineral constraints used in the initial fluid.

\begin{tabular}{|c|c|c|}
\hline Element & Concentration $^{\dagger}$ & Constraint \\
\hline $\mathrm{K}^{+}$ & & K-feldspar \\
\hline $\mathrm{Na}^{+}$ & $10^{-3}$ & \\
\hline $\mathrm{SiO}_{2}$ & & Quartz \\
\hline $\mathrm{CO}_{2}$ & $10^{-4}$ & \\
\hline $\mathrm{Al}^{+++}$ & & Albite \\
\hline $\mathbf{H}^{+}$ & & Muscovite \\
\hline $\mathrm{Cl}^{-}$ & & Charge balance \\
\hline
\end{tabular}

$\dagger$ Total concentration in moles $\mathrm{kg}^{-1}$ 
Table 3: Thermodynamic data used in simulations $\dagger$.

Minerals ${ }^{1}$

\begin{tabular}{|lrrrrrrrr|}
\hline Temp $\left({ }^{\circ} \mathrm{C}\right)$ & 0 & 25 & 60 & 100 & 150 & 200 & 250 & 300 \\
\hline Quartz & -4.61 & -4.03 & -3.47 & -3.07 & -2.73 & -2.46 & -2.20 & -2.03 \\
K-feldspar & -2.63 & -2.51 & -2.75 & -3.32 & -4.12 & -4.79 & -5.32 & -6.00 \\
Albite & 0.13 & -0.21 & -0.97 & -2.00 & -3.22 & -4.20 & -4.97 & -5.81 \\
Kaolinite & 4.92 & 3.17 & 0.89 & -1.44 & -3.94 & -6.01 & -7.79 & -9.50 \\
Muscovite & 11.42 & 8.51 & 4.72 & 0.85 & -3.28 & -6.74 & -9.73 & -12.59 \\
\hline
\end{tabular}

Aqueous Complexes ${ }^{2}$

\begin{tabular}{|lrrrrrrrr|}
\hline Temp $\left({ }^{\circ} \mathrm{C}\right)$ & 0 & 25 & 60 & 100 & 150 & 200 & 250 & 300 \\
\hline $\mathrm{OH}^{-}$ & 14.93 & 13.99 & 13.02 & 12.24 & 11.59 & 11.22 & 11.09 & 11.28 \\
$\mathrm{CO}_{2}(\mathrm{aq})$ & -17.20 & -16.69 & -16.40 & -16.47 & -16.95 & -17.71 & -18.70 & -20.05 \\
$\mathrm{HCO}_{3}^{-}$ & -10.62 & -10.34 & -10.13 & -10.08 & -10.22 & -10.49 & -10.90 & -11.49 \\
$\mathrm{HCl}(\mathrm{aq})$ & 0.80 & 0.86 & 0.83 & 0.70 & 0.42 & 0.04 & -0.48 & -1.24 \\
$\mathrm{KCl}(\mathrm{aq})$ & 2.35 & 2.08 & 1.74 & 1.39 & 0.97 & 0.56 & 0.10 & -0.48 \\
$\mathrm{NaCl}(\mathrm{aq})$ & 0.83 & 0.77 & 0.65 & 0.47 & 0.20 & -0.11 & -0.51 & -1.05 \\
$\mathrm{NaOH}(\mathrm{aq})$ & 15.22 & 14.20 & 13.06 & 12.07 & 11.16 & 10.49 & 9.95 & 9.52 \\
$\mathrm{Al}(\mathrm{OH})^{++}$ & 5.69 & 4.93 & 4.10 & 3.50 & 2.65 & 2.13 & 1.75 & 1.34 \\
$\mathrm{Al}(\mathrm{OH})_{4}^{-}$ & 25.44 & 22.20 & 19.01 & 17.30 & 15.61 & 13.80 & 11.99 & 10.30 \\
$\mathrm{H}_{3} \mathrm{SiO}_{4}^{-}$ & 10.17 & 9.82 & 9.44 & 9.20 & 9.11 & 9.19 & 9.38 & 9.62 \\
\hline
\end{tabular}

1 Thermodynamic data from Sverjensky and others (1991) and Sverjensky (pers. commun.). 2 Thermodynamic data from Wolery (1983).

$\dagger$ Reactions written in terms of primary species $\mathrm{H}^{+}, \mathrm{H}_{2} \mathrm{O}, \mathrm{K}^{+}, \mathrm{Na}^{+}, \mathrm{Al}^{+++}, \mathrm{SiO}_{2(a q)}, \mathrm{CO}_{3}^{--}$, and $\mathrm{Cl}^{-}$in all cases as the destruction of 1 mole of the mineral or secondary species. For example, the reaction for $\mathrm{K}$-feldspar would be written as

$\mathrm{KAlSi}_{3} \mathrm{O}_{8}+4 \mathrm{H}^{+}=\mathrm{K}^{+}+\mathrm{Al}^{+++}+\mathrm{SiO}_{2, a q}+2 \mathrm{H}_{2} \mathrm{O}$ 
Table 4: Estimates of fracture spacing, fracture aperture, and flow porosities in natural environments.

\begin{tabular}{llll}
\hline Flow porosity (\%) & Fracture aperture $(\mathrm{m})$ & Fracture spacing $(\mathrm{m})$ & Ref. \\
\hline $5 \times 10^{-6}$ to 1 & $5 \times 10^{-7}$ to $2 \times 10^{-4}$ & 10 to 0.22 & 1 \\
0.029 & $6.4 \times 10^{-5}$ & 0.22 & 2 \\
0.285 & $1 \times 10^{-4}$ & 0.035 & 3 \\
0.016 & $1 \times 10^{-4}$ & 0.625 & 4 \\
0.182 & $1 \times 10^{-4}$ & 0.055 & 5 \\
\hline
\end{tabular}

1. Norton and Knapp (1977)

2. Pruess and others (1990)

3. Average of 6 horizontal veins, Skaergaard Intrusion-Manning and Bird (1991)

4. Average of 16 vertical pyroxene veins, Skaergaard Intrusion-Manning and Bird (1991)

5. Average of 22 vertical amphibole veins, Skaergaard Intrusion-Manning and Bird (1991)

Table 5: Initial physical parameters used in simulations with reaction-induced porosity-permeability change.

\begin{tabular}{lll}
\hline Flow porosity (\%) & Fracture aperture $(\mathrm{m})$ & Fracture spacing $(\mathrm{m})$ \\
\hline 1 & $1.9 \times 10^{-6}$ & 0.00019 \\
0.1 & $5.9 \times 10^{-6}$ & 0.0059 \\
0.01 & $1.9 \times 10^{-5}$ & 0.19 \\
\hline
\end{tabular}


Table 6: Reaction rate constants at $25^{\circ} \mathrm{C}$ and activation energies used in the simulations.

\begin{tabular}{lccc}
\hline Mineral & $\begin{array}{c}k_{25^{\circ} \mathrm{C}} \\
\left(\mathrm{moles} \mathrm{m}^{-2} \mathrm{~s}^{-1}\right)\end{array}$ & $\begin{array}{c}\text { Activation Energy } \\
\left(\mathrm{kJ} \mathrm{mol}^{-1}\right)\end{array}$ & Reference \\
\hline Quartz & $4.30 \times 10^{-14}$ & 75.0 & 1 \\
K-feldspar & $3.09 \times 10^{-12}$ & 38.2 & 2 \\
Albite & $3.09 \times 10^{-12}$ & 38.2 & 3 \\
Kaolinite & $2.36 \times 10^{-14}$ & $62.8^{\dagger}$ & 4 \\
Muscovite & $2.36 \times 10^{-14}$ & $62.8^{\dagger}$ & 5 \\
\hline
\end{tabular}

$\dagger$ Activation energy assumed (Lasaga, 1984)

1. Rimstidt and Barnes (1980)

2. Helgeson and others (1984)

3. Chosen to be the same as K-feldspar

4. Rate from $80^{\circ} \mathrm{C}$ data of Nagy and others (1990)

5. Chosen to be the same as kaolinite

Table 7: Maximum calculated precipitation rates of quartz and Kfeldspar for Rayleigh numbers of 50, 100, 200, and 300 .

\begin{tabular}{llccr}
\hline $\begin{array}{l}\text { Rayleigh } \\
\text { Number }\end{array}$ & Mineral & $\begin{array}{c}\text { Maximum rate } \\
\left(\text { moles } \mathrm{m}^{-3} \mathrm{yr}^{-1}\right)\end{array}$ & $\begin{array}{r}\text { Maximum volume \% change } \\
\left(\mathrm{yr}^{-1}\right)\end{array}$ & $\begin{array}{r}\text { Years to change } \\
\text { volume by 1\% }\end{array}$ \\
\hline 50 & Quartz & $5.0 \times 10^{-5}$ & $1.1 \times 10^{-7}$ & $9,090,907$ \\
& K-feldspar & $1.9 \times 10^{-6}$ & $2.1 \times 10^{-8}$ & $47,619,047$ \\
100 & Quartz & $7.0 \times 10^{-4}$ & $1.6 \times 10^{-6}$ & 625,000 \\
& K-feldspar & $2.6 \times 10^{-5}$ & $2.8 \times 10^{-7}$ & $3,571,428$ \\
200 & Quartz & $1.5 \times 10^{-3}$ & $3.4 \times 10^{-6}$ & 294,117 \\
& K-feldspar & $6.4 \times 10^{-5}$ & $7.0 \times 10^{-7}$ & $1,428,571$ \\
300 & Quartz & $2.3 \times 10^{-3}$ & $5.2 \times 10^{-6}$ & 192,307 \\
& K-feldspar & $9.5 \times 10^{-5}$ & $1.0 \times 10^{-6}$ & $1,000,000$ \\
\hline
\end{tabular}




\subsection{Effect of Rayleigh Number on Mineral Reaction Rates}

The Rayleigh number (Equation 33) provides the most convenient single parameter against which to compare the magnitude and spatial distribution of mineral precipitation and dissolution reactions in a hydrothermal system. This is done in this section by carrying out transient calculations of coupled chemical reaction, heat transfer, mass transport, and fluid flow using the numerical scheme, boundary, and initial conditions described above. In these simulations, however, the porosity and permeability are held constant. K-feldspar is assumed to make up $39 \%$ of the rock by volume, with quartz and albite both accounting for $30 \%$ each, resulting in a porosity of $1 \%$. Permeabilities of $0.73,1.46,2.92$, and 4.37 millidarcies are used to obtain Rayleigh numbers of 50, 100, 200, and 300 respectively. These permeabilities are well within the range reported for many fractured rocks where measured on the crustal scale (e.g., Brace, 1984; Clauser, 1992). In these simulations, the computational width of the system is 2000 meters which is discretized using a constant 100 meter grid spacing in both vertical and horizontal directions. The additional 2000 meters width shown in Figure 11 through Figure 13 is obtained by symmetry. The initial time step is about 1 second and is gradually increased to a maximum of 5 years.

Figure 11 shows the temperature fields corresponding to steady-state convection for Rayleigh numbers of $50,100,200$, and 300 . For a Rayleigh number of 50 , the convection causes only a very slight disturbance in the isotherms, indicating that heat transfer is dominated by conduction. In contrast, at a Rayleigh number of 300 a boundary layer has developed near the no-flux top of the system and the central portion of the upwelling plume shows only a small temperature change (from $300^{\circ} \mathrm{C}$ to $250^{\circ} \mathrm{C}$ over 1500 meters). The corresponding Darcy fluxes are shown in Figure 12 (except for $\mathrm{Ra}=50$, where the fluxes are too small to be shown when the same scaling is used) with a representative vector length of $0.5 \mathrm{~m} \mathrm{yr}^{-1}$ shown below the $\mathrm{Ra}=300$ cell.

Quartz precipitation rates for the steady-state convection cells are shown in Figure 13. Contour intervals of $1 \times 10^{-4}$ moles $\mathrm{m}^{-3} \mathrm{yr}^{-1}$ are used and in every case, the contour label is placed on the lower side. Note that the most intense quartz precipitation occurs in the thermal boundary layer developed near the top of the system. A significant amount of quartz also precipitates at about 1300 meters depth where the change in the solubility of the quartz as a function of temperature is the greatest and where the fluid fluxes reach a maximum value. The negative side of the 0 rate contour corresponds to a region where quartz is dissolving (not shown here).

As the significance of an instantaneous precipitation rate presented in units of moles $\mathrm{m}^{-3} \mathrm{yr}^{-1}$ may be unclear, it may be easier to visualize if converted to a change in the volume percent of quartz per unit time (see Equation 26 and Equation 27). Table 5 gives the maximum calculated precipitation rates along with the rates of change of the volume percents for quartz and K-feldspar at the various Rayleigh numbers considered. Note that according to the calculations, at a Rayleigh number of 300 it would take about 192,000 years to add 1 volume $\%$ of quartz to the rock where the precipitation rate is at its highest. 


\section{Temperature}
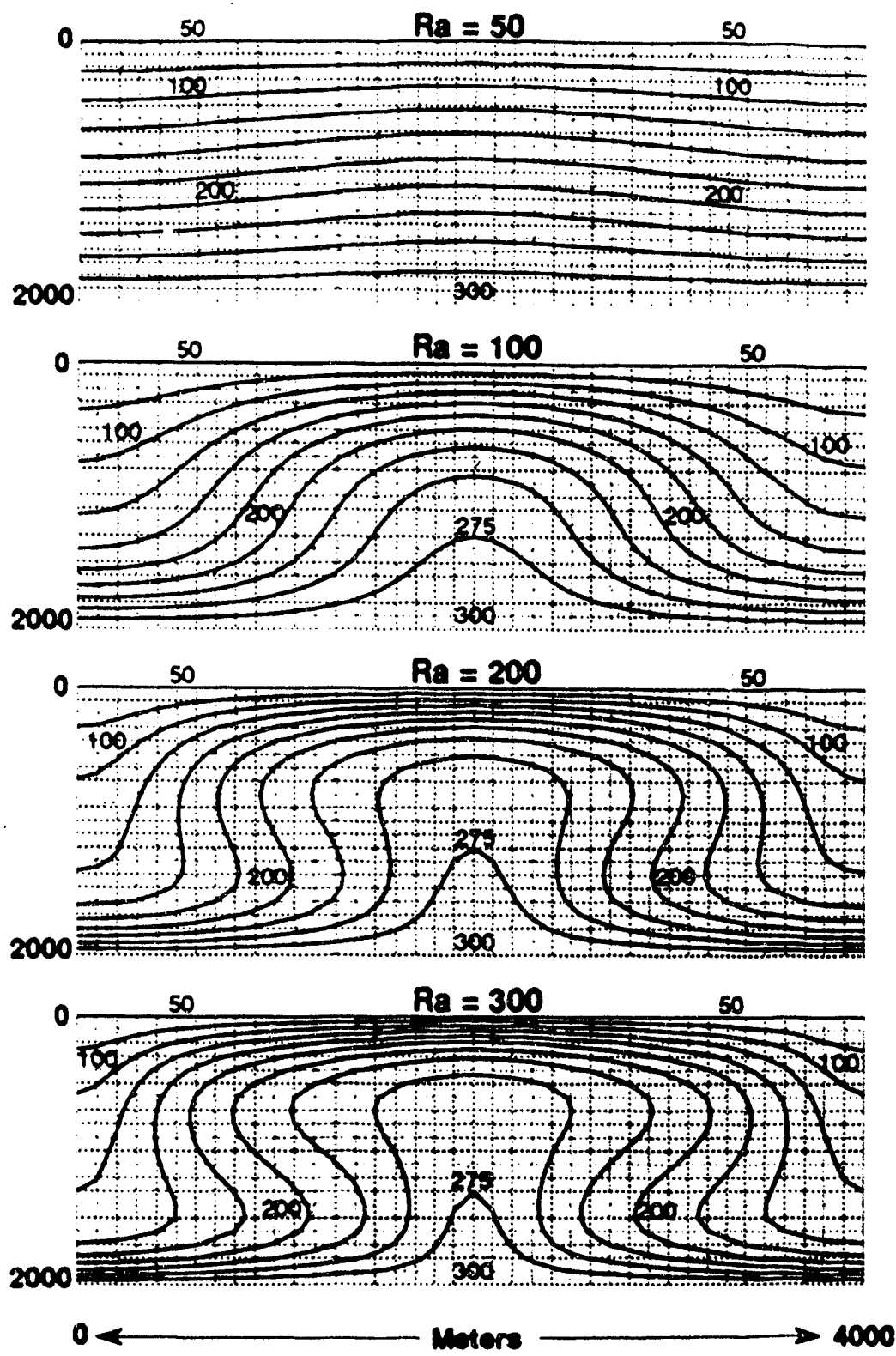

Figure 11 Temperature field in steady-state hydrothermal convection cell for Rayleigh ( $\mathrm{Ra}$ ) numbers of $50,100,200$, and 300 . Contour intervals in $25^{\circ} \mathrm{C}$ increments. Computational grid with uniform 100 meter spacings shown as light gray. 

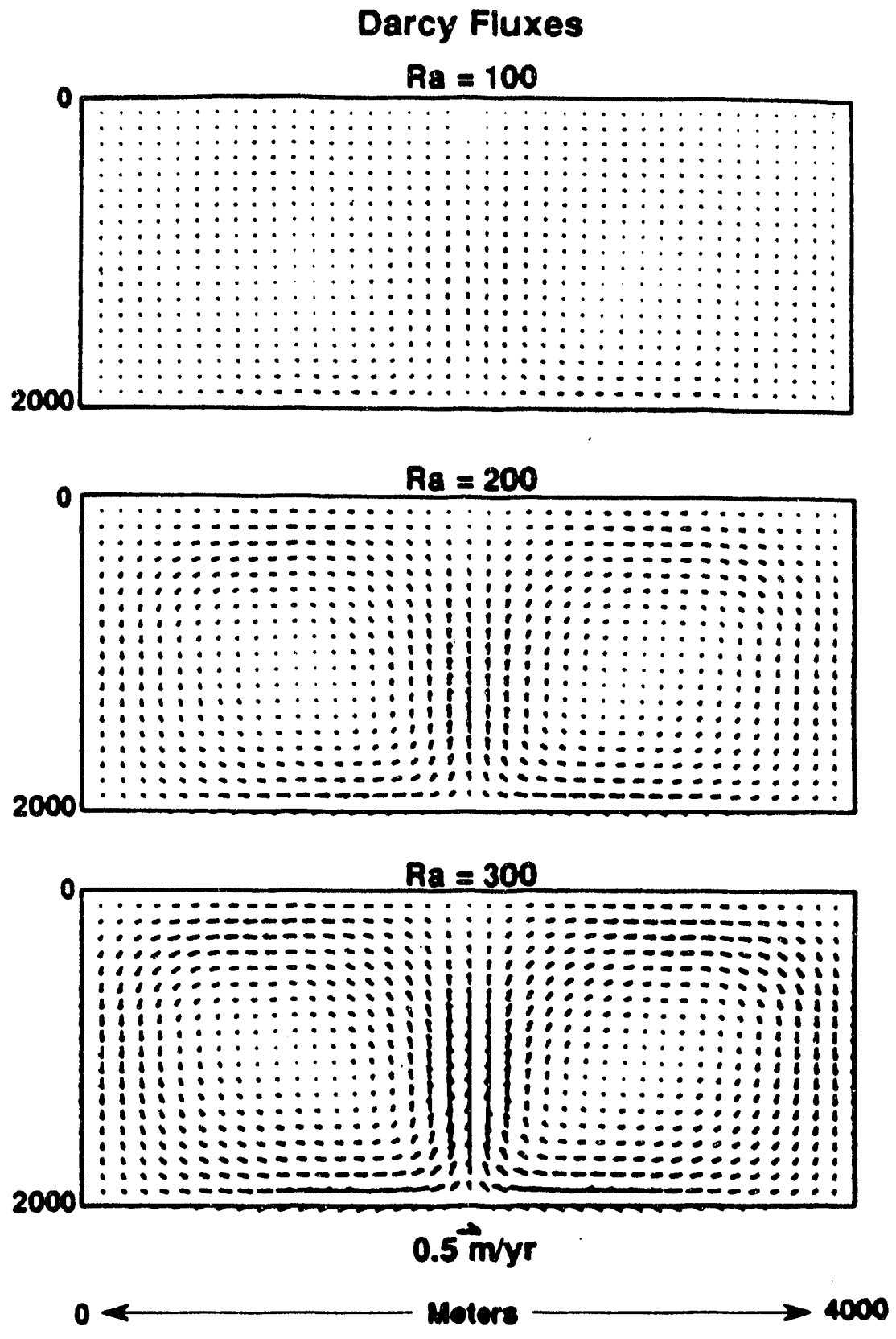

Figure 12 Darcy fluxes in steady-state hydrothermal convection cells for Rayleigh numbers ( $\mathrm{Ra}$ ) of 100,200 , and 300 . A Darcian flux of $0.5 \mathrm{~m}^{3} \mathrm{~m}^{-2} \mathrm{yr}^{-1}$ corresponds to the vector length plotted below the $\mathrm{Ra}=300$ cell. The same scaling of vector lengths is used throughout. 


\section{Quartz Precipitation Rate}
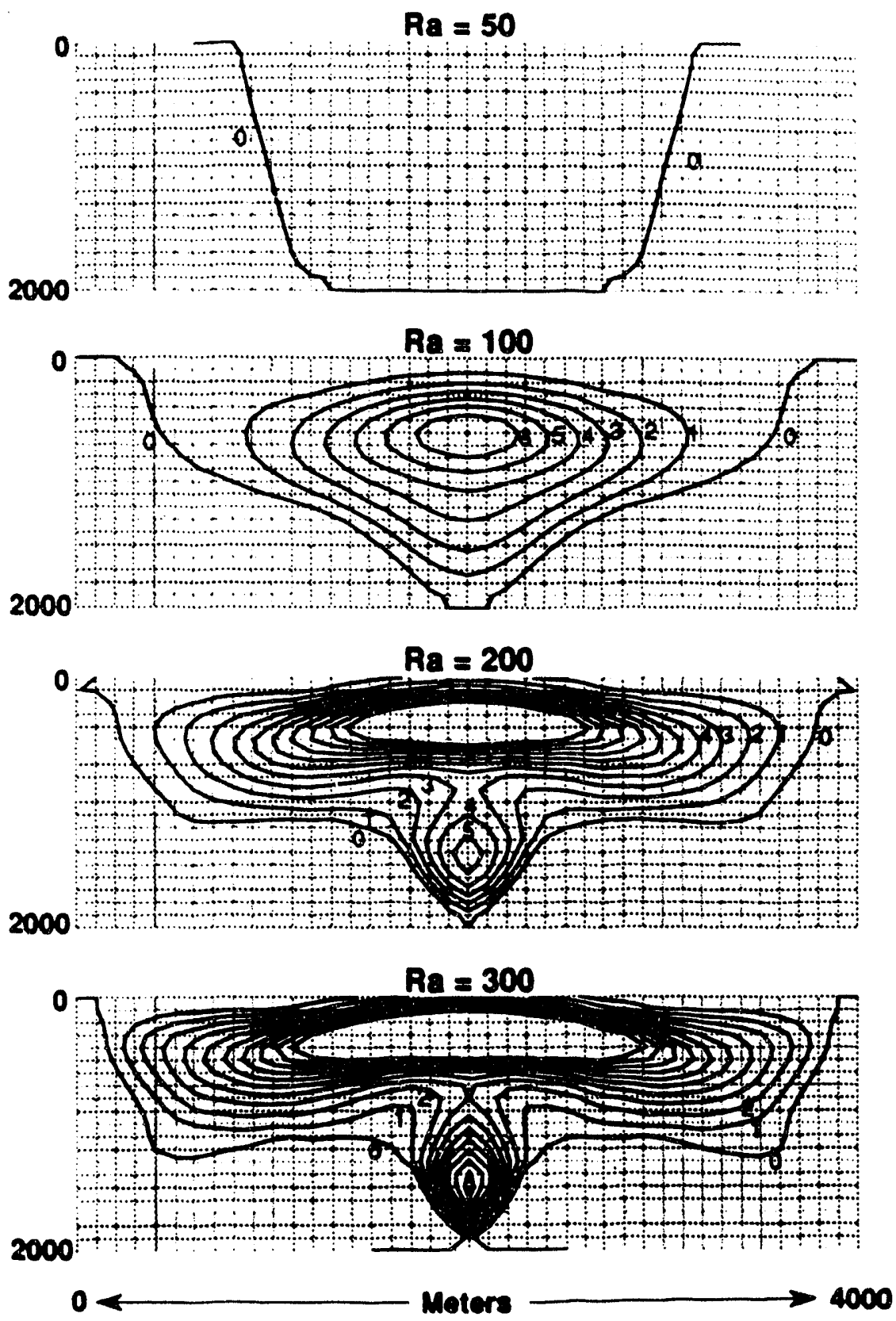

Figure 13 Calculated quartz precipitation rates in steady-state convection cells for Rayleigh ( $\mathrm{Ra}$ ) numbers of $50,100,200$, and 300 . Contour increments are $1 \times 10^{-4}$ moles $\mathrm{m}^{3} \mathrm{yr}^{-1}$ (i.e., labelled contours have been multiplied by $1 \times 10^{4}$ ). 
$1,000,000$ years would be required to add that much $\mathrm{K}$-feldspar, but this result reflects the dilute character of the hydrothermal system. The results for quartz, in contrast, don't depend greatly on the initial conditions since the concentration of aqueous silica is largely buffered by quartz. It is also important to point out that these numbers represent the volume added uniformly to the large area corresponding to the elemental volume of $100 \mathrm{~m}$ by $100 \mathrm{~m}$ considered here. Obviously if this total amount of silica were added to a much more localized region, as in a series of discrete fractures, then the local volume of quartz precipitated would be many times larger. This points out the importance of eventually being able to treat more strongly heterogeneous porous formations.

\subsection{Effect of Permeability Change on the Transient Evolution of a Convection Cell}

The simulations presented above do not include feedback between the precipitation/dissolution reactions and the permeability of the rock. We wish to consider here the question of how the time scales for reaction-induced permeability change compare to the characteristic time scales of the convection process itself. One way to approach this problem would be to carry out simulations of a cooling pluton, as in the fashion of Norton and Knight (1977), and compare the computed reaction-induced changes with the normal time scales required for the thermal anomaly caused by the emplacement of a pluton to dissipate. So as to avoid the necessity of treating chemical reactions above $300^{\circ} \mathrm{C}$ (the upper limit of the present database), we have chosen to compare the integrated rates of permeability change with the time required for a hydrothermal convection cell with a constant-temperature lower boundary to achieve a steady state. We focus here on a hydrothermal system with a Rayleigh number of 200 and use all of the same physical and parameters as listed in Table 1 and the same initial chemical conditions as given in Table 2. As a variable we consider only the effect of changing the flow porosity, $\phi_{F}$, which is defined below.

As pointed out by Budi Sagar (pers. communication, 1992), the presence of no flux boundary conditions can have a significant effect on the dynamical behavior of the system where permeability change occurs. In the Rayleigh number simulations described above, the no flow boundaries were placed 4000 meters apart, which corresponds to the natural wavelength predicted by linear stability analysis for a system which is 2000 meters deep (Turcotte and Schubert, 1982). Once the permeability distribution evolves in space because of the mineral precipitation and dissolution reactions, however, the presence of a prescribed no flux boundary may impose a certain behavior on the system. Accordingly, in the horizontal dimension we have increased the width of the computational domain from 2000 meters to 8000 meters, making no assumptions of a symmetry boundary within that distance. A constant grid spacing of 200 meters is used in the horizontal direction while the vertical spacing remains at 100 meters. The upper and lower boundaries are still assumed to be no flux. Geologically, one could think of this as a case where a relatively permeable formation (of initially 2.92 millidarcies) is sandwiched between two virtually 
impermeable rocks (e.g., a shale). It is important to point out, however, that a system in which the initial permeability decreased slowly with depth would show significantly different behavior from a system in which a no flux boundary is imposed.

\subsection{Porosity-Permeability Model for Fractured Rock}

In order to couple the porosity changes due to chemical reactions calculated from Equation 27 and Equation 28, we need a model which relates the porosity of the medium to its permeability. The simplest possible model for fractured media is that of a set of parallel fractures with smooth walls (e.g., Snow, 1970; Norton and Knapp, 1977; Marsily, 1986; Pruess and others, 1990). In this model, one defines a flow (also referred to as the fracture or kinematic) porosity by

$$
\phi_{F}=\frac{\delta}{d}
$$

where $\delta$ is the fracture aperture and $d$ is the fracture spacing. This formulation also provides an expression for the rock permeability

$$
k=\frac{\delta^{3}}{12 d}
$$

or written in terms of the flow porosity,

$$
k=\frac{d^{2} \phi_{F}^{3}}{12} .
$$

From this porosity-permeability relationship, we can calculate the reaction-induced changes in rock permeability as a function of time if a number of simplifying assumptions are made. The most important of these is that the changes in mineral volumes in the rock due to reaction take place entirely along the fracture walls (i.e., not within the rock matrix where presumably transport is by diffusion). Although in general this assumption is not really valid for many systems, a simultaneous treatment of flow in fractures and matrix diffusion is beyond the scope of the present study. The calculations are useful in showing the behavior of the system in the limiting case where rock diffusion is relatively minor. If we assume that the fracture spacing in the rock is constant with time, then differentiating Equation 36 with respect to time and making use of Equation 34 gives

$$
\frac{\partial k}{\partial t}=\left(\frac{\delta^{2}}{4}\right) \frac{\partial \phi_{F}}{\partial t},
$$

which indicates that the magnitude of the permeability change for a given change in the rock's flow porosity is determined by $\delta$, the fracture aperture. A second assumption which is made is that there is no distribution of fracture apertures (i.e., within any one elemental volume they are assumed to be all of the same size). As the porosity and permeability 
evolves in the simulations, however, variations in fracture aperture develop in space. Additionally, we assume that the fractures retain smooth, parallel walls throughout the course of the simulation.

\subsection{Porosity-Permeability Ranges in Fractured Rocks}

There have been a number of attempts to estimate porosity-permeability relationships in fractured rocks. Norton and Knapp (1977), using a planar fracture model of the kind described above, estimated that flow porosities had a total range between $1 \%$ and $5 \times 10^{-6} \%$, although the more common range they observed was between $0.1 \%$ and $0.001 \%$. These estimates are derived from their measurements of the fracture density (or fracture spacing) and fracture apertures which are summarized in Table 4. Pruess and others (1990), citing studies by Scott and others (1982) and Peters and others (1984), report that the fracture spacing at the Yucca Mountain site in Nevada is on the order of 0.3 meters and fracture apertures range from about 10 to $100 \mu \mathrm{m}$. Manning and Bird (1991) measured cumulative vein densities (i.e., $d^{-1}$ or $n$ in the terminology of Norton and Knapp [1977]) in the contact aureole of the Skaergaard intrusion in Greenland and found that the fracture apertures ranged from about $0.1 \mathrm{~mm}$ to $1 \mathrm{~mm}$ with the more common value being closer to $0.1 \mathrm{~mm}$. A value of $0.1 \mathrm{~mm}$ is used in the calculations of the flow porosity in Table 4. Note that Manning and Bird (1989) actually give the porosities as simple fractions and not as percents. Their numbers, therefore, should be multiplied by 100 in order to express the porosity as a percent (see their Equation 3).

\subsection{Transient Evolution of Systems without Permeability Change}

As a basis for comparison, we examine here the transient evolution of a system in which precipitation and dissolution reactions have no effect on the rock's porosity and permeability. The temperature field is shown in 5,000 year intervals between 10,000 and 30,000 years in Figure 14. From the figure it is apparent that the system reaches a quasi-steady state between 25,000 and 30,000 years. The hydrothermal system by this time has settled in to a stable configuration in which downwelling regions at 2,000 and 6,000 meters define a convection cell with a wavelength of 4,000 meters. Following the evolving temperature and flow field, solution $\mathrm{pH}$, which is largely determined by the initial conditions and near-equilibrium between albite and K-feldspar, evolves to the geometries shown in Figure 15. The quartz precipitation rate evolves gradually as well (Figure 16) so that by 30,000 years its geometry coincides with that shown in Figure 13. Both the solution $\mathrm{pH}$ and the quartz precipitation rate (along with all the other mineral dissolution and precipitation rates) will maintain this geometry until either one of the primary minerals dissolves completely (or nearly so in the kinetic case) at some point in space or until some other factors act to change the temperature and flow field. These factors could, of course, be externally imposed effects, like the cooling of the underlying heat source, but in 

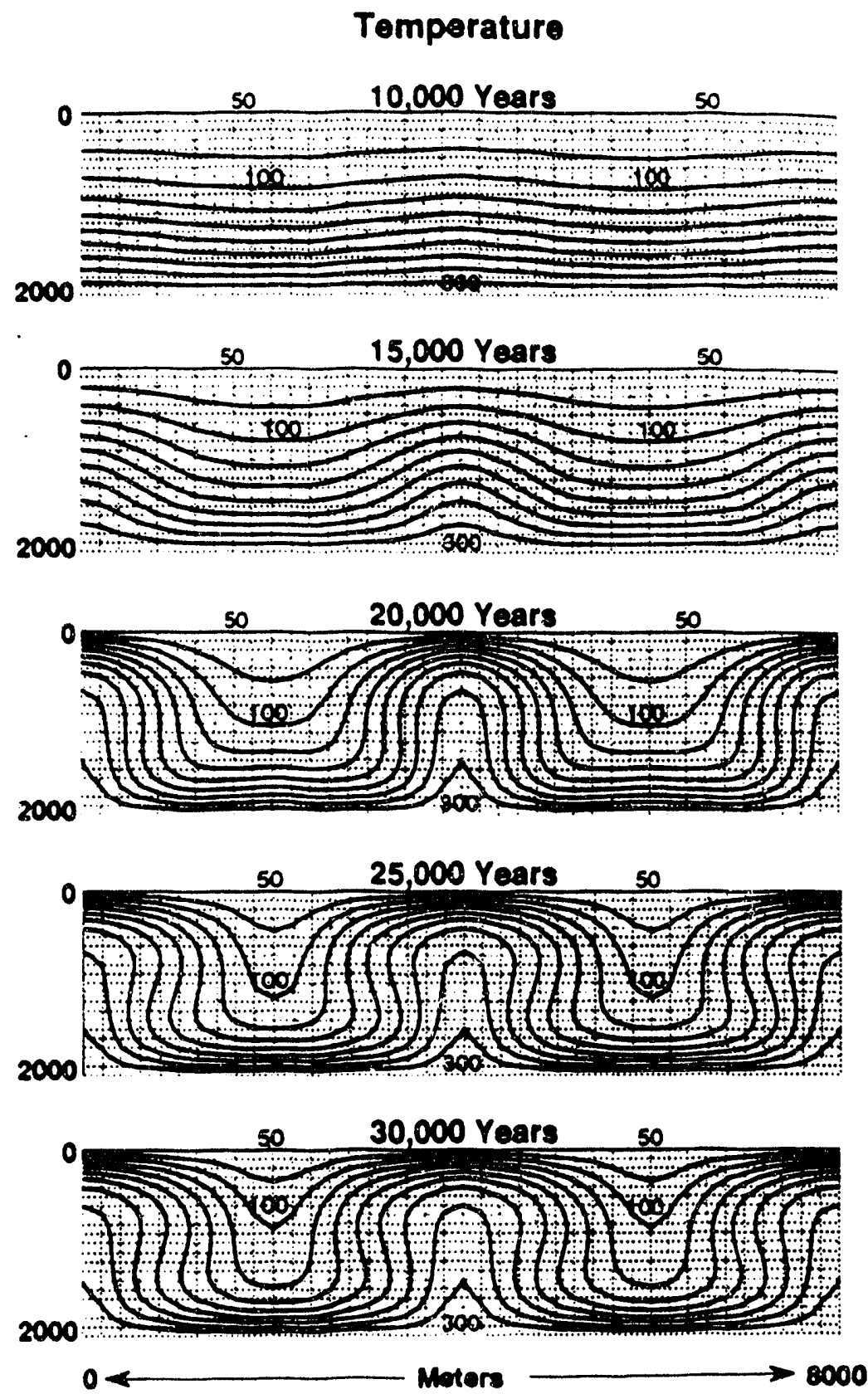

Figure 14 Transient evolution of a temperature field in a hydrothermal system characterized by a Rayleigh number of 200 without changes in porosity and permeability. Grid spacing is 200 meters in the horizontal direction and 100 meters in the vertical (shown as pale gray). 

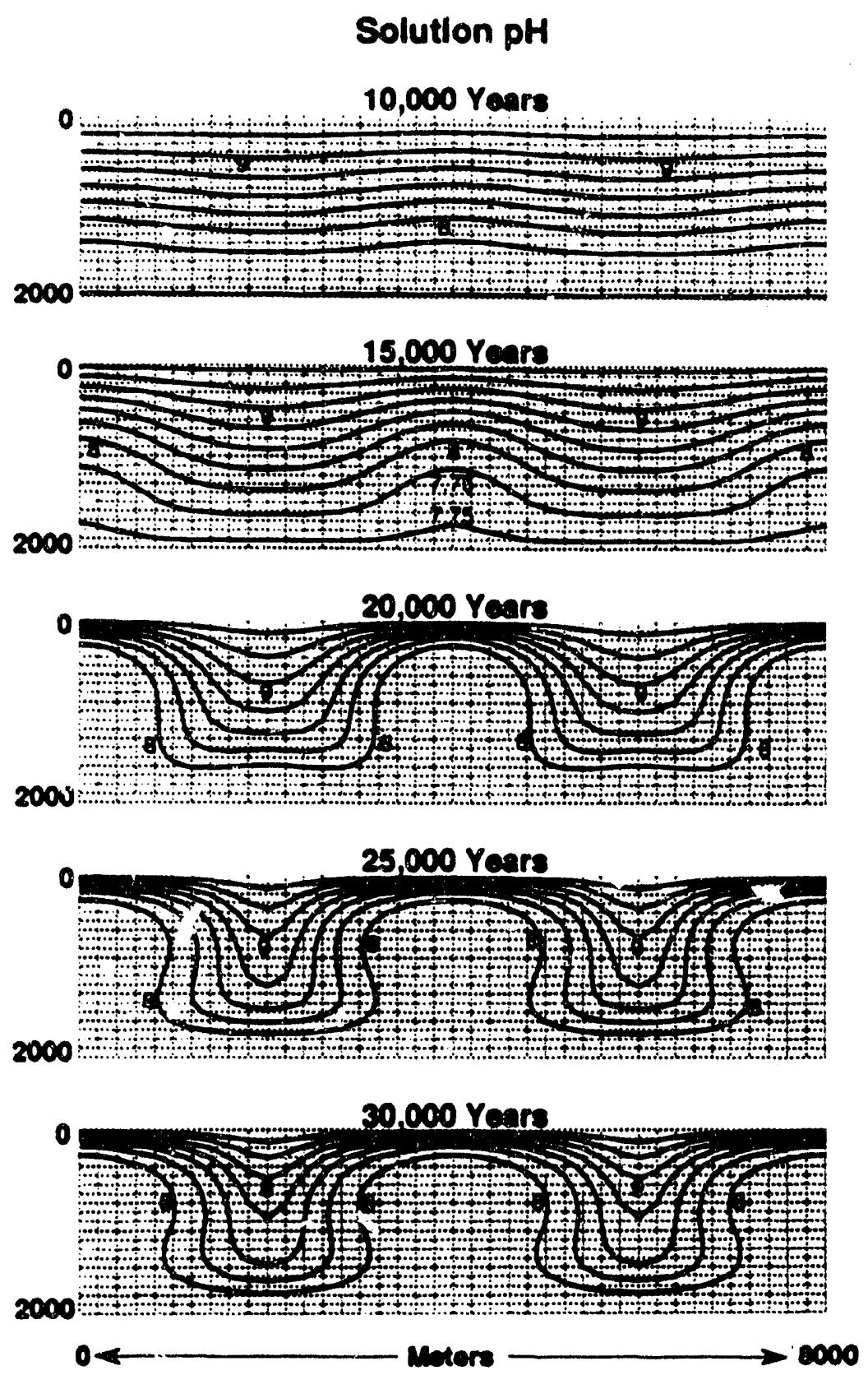

Figure 15 Transient evolution of solution $\mathrm{pH}$ in a $\mathrm{Ra}=200$ convection cell with no changes in porosity and permeability included. Contour increments are in increments of $3.25 \mathrm{pH}$ units. 

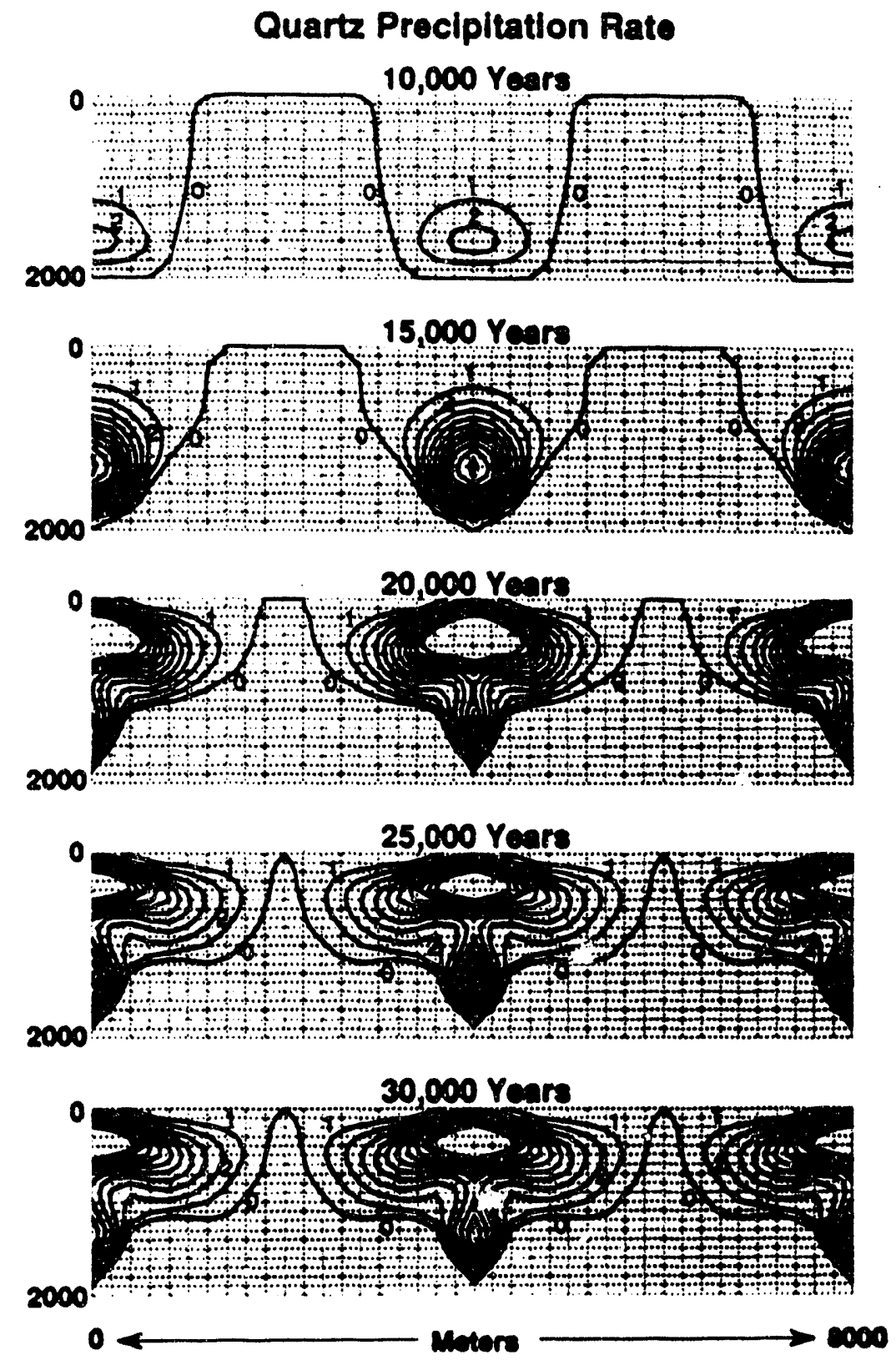

Figure 16 Transient evolution of quartz precipitation rates in a $R_{a}=200$ convection cell with no changes in porosity and permeability considered. Contour increments are $1 \times 10^{-4}$ moles $\mathrm{m}^{3} \mathrm{yr}^{-1}$ (i.e., labelled contours are multiplied by $1 \times 10^{4}$ ). 
the section that follows we examine the self-organizational effect of permeability changes caused by the mineral reactions themselves.

\subsection{Simulations with Permeability Change}

Based on the estimates of flow porosities given in Table 4, it would appear that the appropriate range of flow porosities to test in the numerical simulations is between about $1 \%$ and $0.01 \%$. Assuming an initial permeability throughout of 2.92 millidarcies (resulting in an initial Rayleigh number of 200), these initial flow porosities yield the physical parameters summarized in Table 5 if Equation 35 and Equation 36 are solved. A comparison of the calculated permeability distributions (in millidarcies) after an elapsed time of 50,000 years is shown in Figure 17 for the cases where the initial flow porosities are $1 \%$ and $0.1 \%$. Note that the simulation which began with a flow porosity of $1 \%$ shows only a very slight change from the initial value of 2.92 millidarcies. In contrast, the simulation which used an initial flow porosity of $0.1 \%$ shows much more dramatic behavior, with a substantial region developing permeabilities greater than 10 millidarcies. Unfortunately, where the initial porosity was assumed to be $0.01 \%$ the simulation became very difficult to carry out numerically due to the extreme permeability change and to the low porosities in the rock, so no results are shown. Accordingly, we will restrict ourselves to analyzing the simulation in which the initial flow porosity was taken to be $0.1 \%$.

In order to grasp the dynamic evolution of the system here, Figure 18 through Figure 23 should be viewed together because of the coupled nature of the processes at work. Figure 18 shows the evolution of the porosity between 10,000 and 50,000 years. Note that the porosity increases dramatically in a highly localized area along the lower boundary of the system due to the dissolution of quartz (Figure 19). This region corresponds to a portion of the system where the fluid is moving up temperature. Because the solubility of quartz is highest at this point in the system, the dissolution rates here are higher than elsewhere. The resulting increase in porosity in this region causes the permeability to increase as well (Figure 20) which in turn leads to a focusing or channeling of the flow field (Figure 21). In contrast, where quartz precipitation causes a reduction in the permeability of the rock, the opposite effect occurs (Figure 22). The permeability reduction has the effect of making the flow more diffuse, that is, the flow field diverges around the low permeability zone. This causes the upwelling plume, which is normally more focused than the recharge zone, to broaden gradually with time. This is shown best by viewing the evolution of the temperature field (Figure 23). Note that the more or less normal convection cell geometry at about 20,000 years gives way gradually to a different shape defining the focused recharge zone and the diffuse upwelling zone.

Because the channeling effect caused in this example by the dissolution of quartz represents an instability due to the positive feedback between the chemical reactions and the flow field, the rate at which the porosity and permeability increase increases with time. 


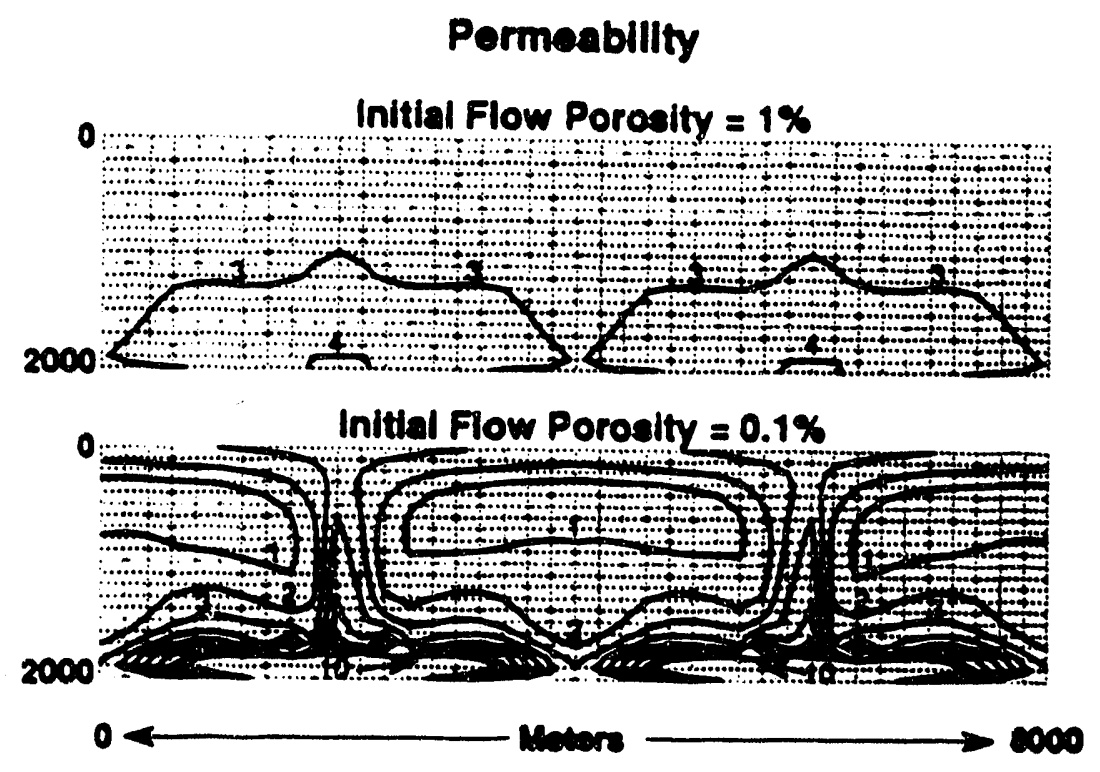

Figure 17: Comparison of calculated permeability distribution (in millidarcies) at 50,000 years for simulations which began with flow porosities of $1 \%$ and $0.1 \%$. 

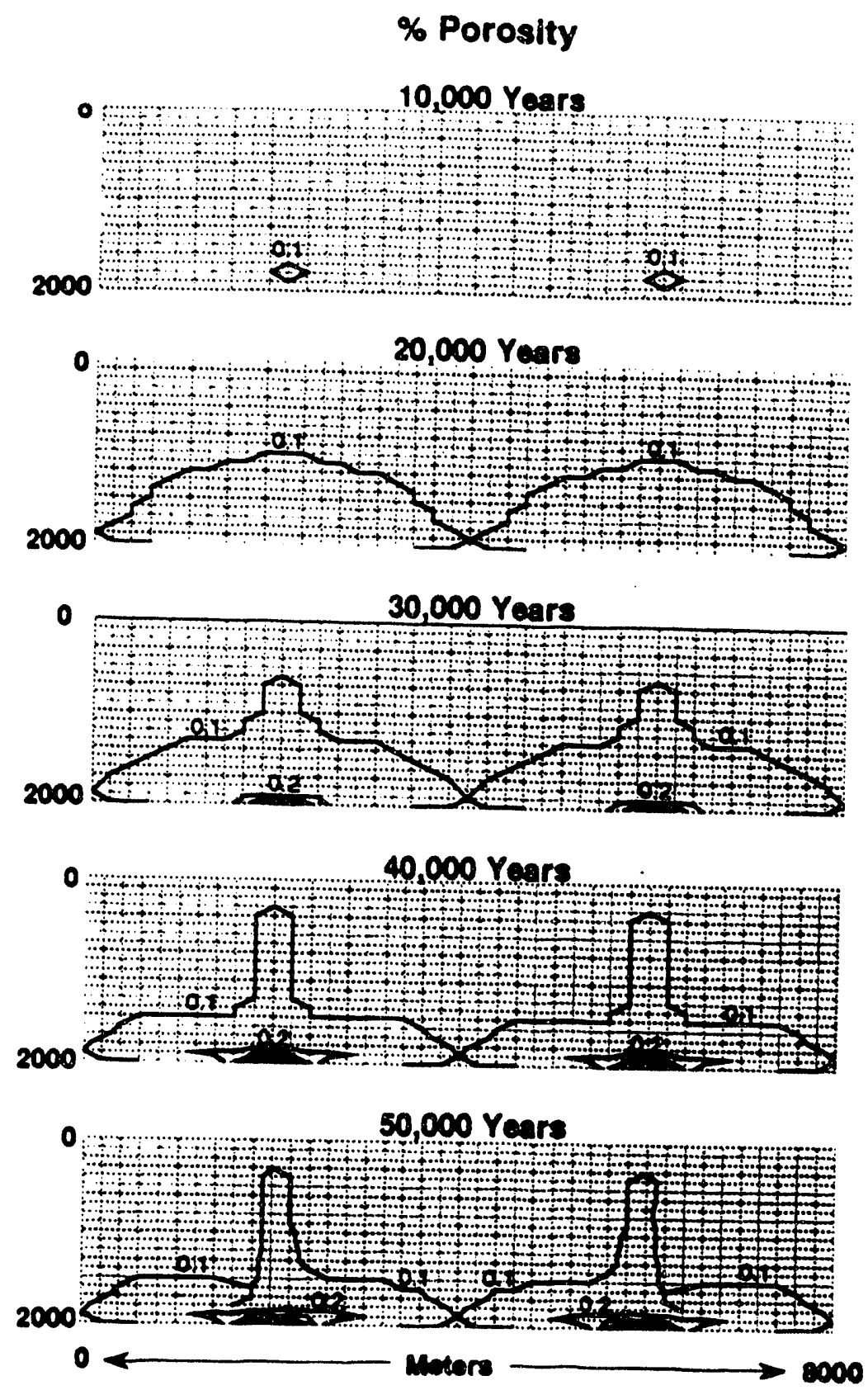

Figure 18: Time evolution of the porosity for the case in which the initial porosity $=0.1 \%$. Contour increments are $0.1 \%$. 

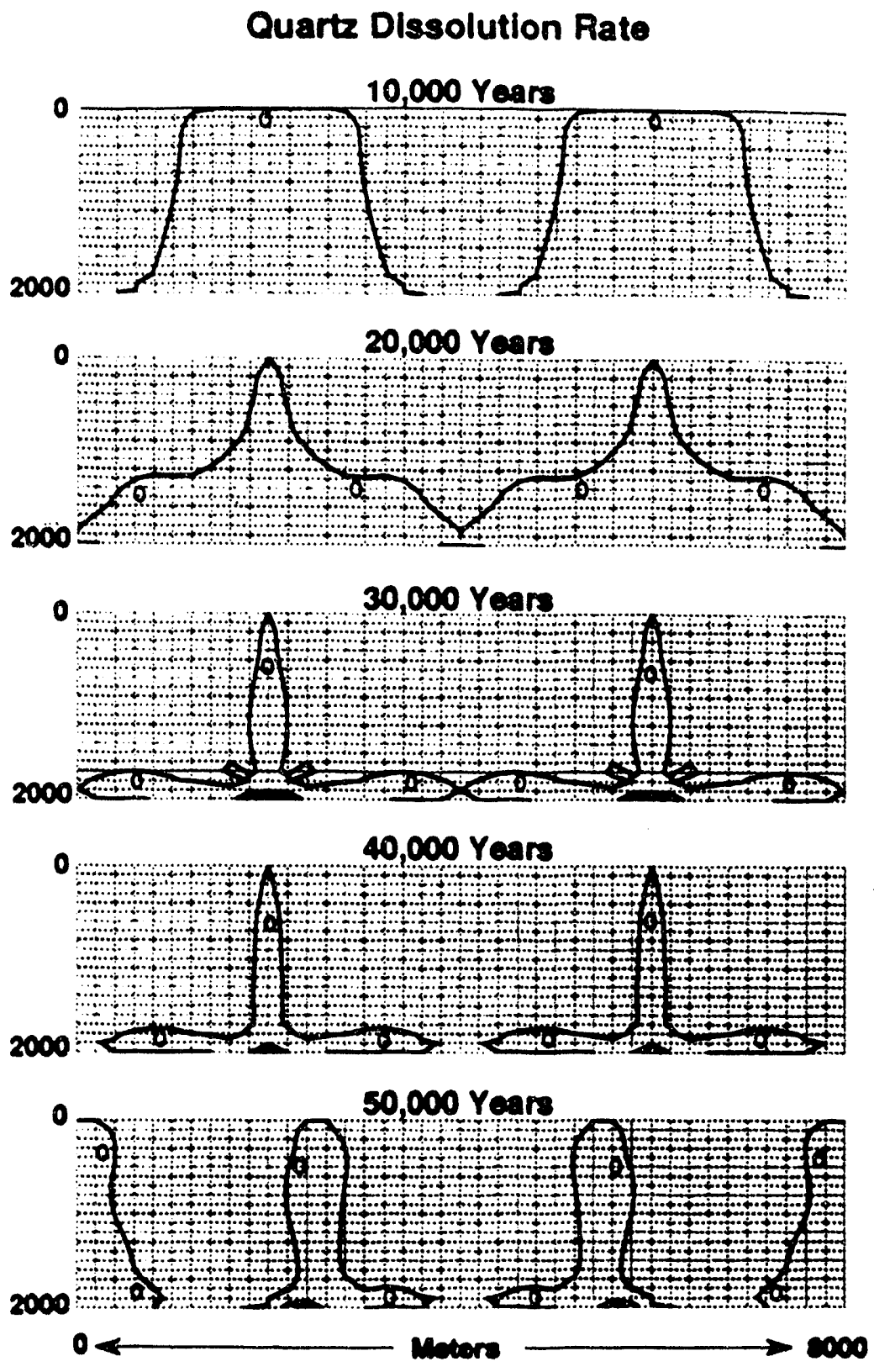

Figure 19: Time evolution of the quartz dissolution rate for the case in which the initial tlow porosity $=0.1 \%$. Contour increments are $1 \times 10^{-4}$ moles $\mathrm{m}^{-3} \mathrm{~s}^{-1}$. Note the highly focused quartz dissolution zone at the base of the convection cell which develops because of the channeling effects. 

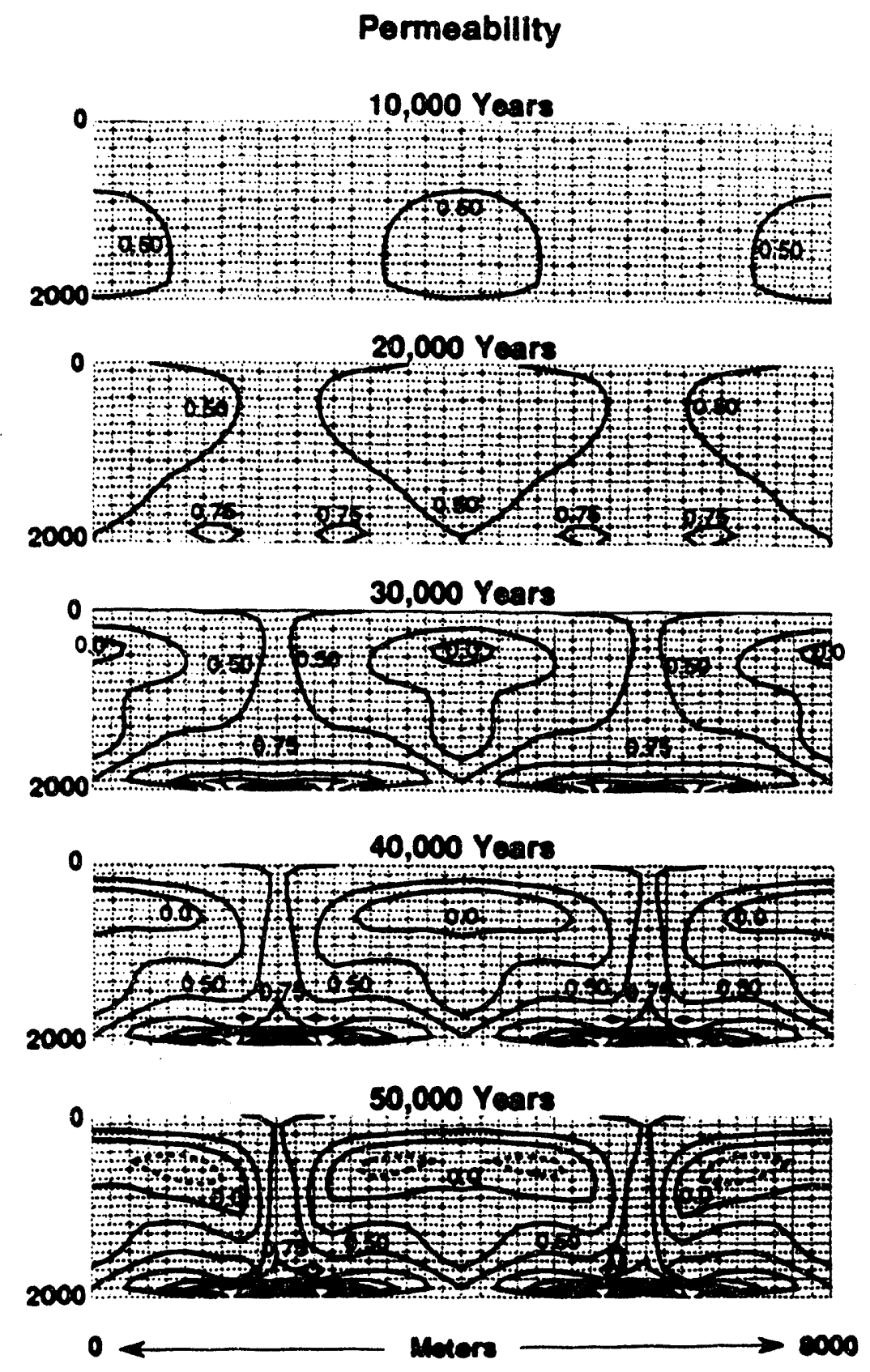

Figure 20: Time evolution of the permeability distribution for the case in which the initial flow porosity $=0.1 \%$. Contour increments are $0.25 \log$ millidarcies. 

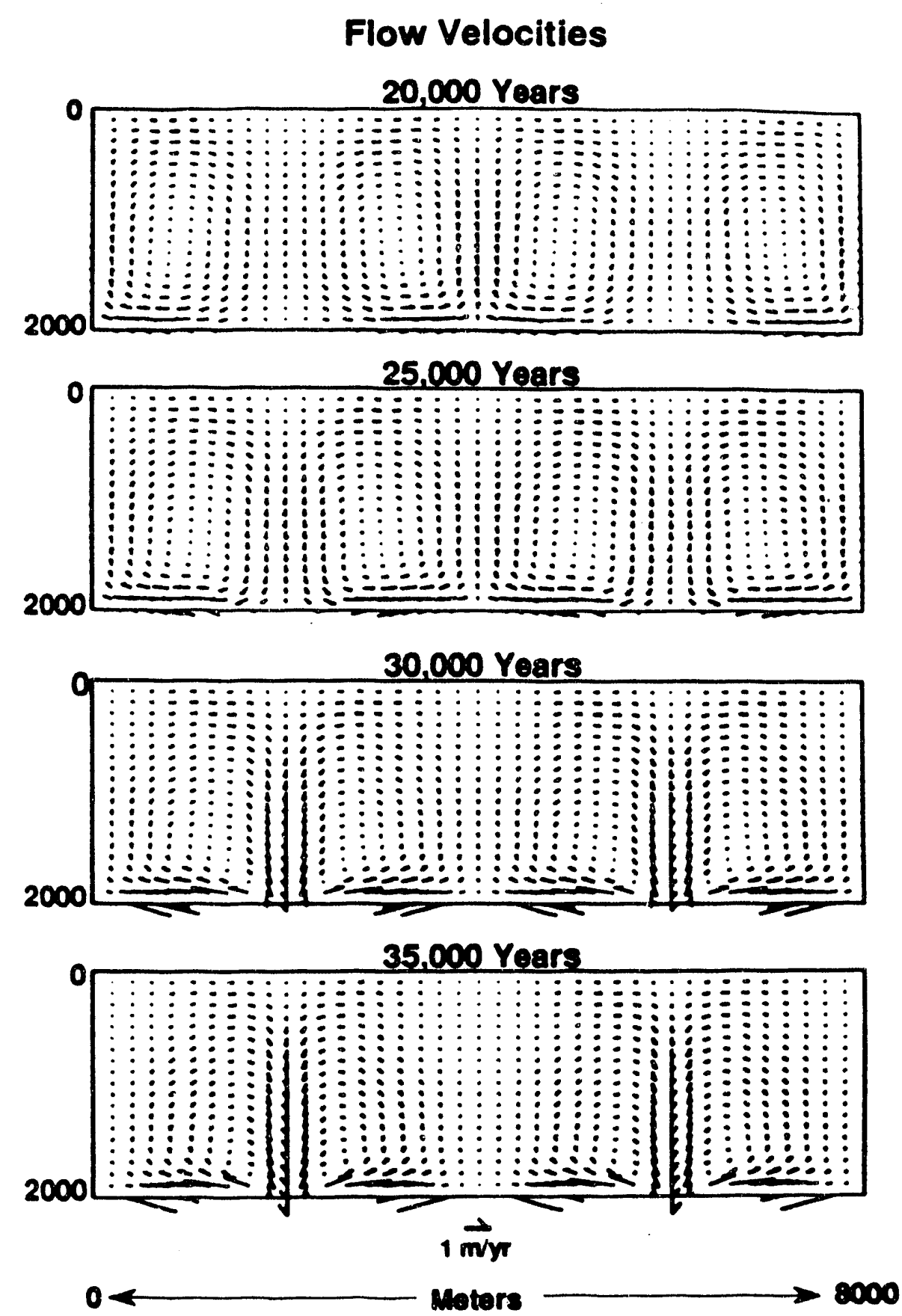

Figure 21: Time evolution of the Darcian flux for the case in which the initial flow $=0.1 \%$. The vector plotted below the contour plot at 35,000 years corresponds to a flux of $1 \mathrm{~m}^{3} \mathrm{~m}^{-2} \mathrm{yr}^{-1}$. All plots use the same scaling. 


In other words, one cannot simply extrapolate the initial rate of porosity change through time and arrive at a reasonable estimate of the integrated porosity and permeability change. The nonlinear process taking place in the simulations is essentially that which was described by Ortoleva and others (1987) and Steefel and Lasaga (1990), except that it is operating here in a nonisothermal environment. In the isothermal example considered by Ortoleva and others (1987) and Steefel and Lasaga (1990), the reactions are driven by the infiltration of a fluid which is out of equilibrium with the rock. That is, the channeling process depends directly on the boundary conditions imposed on the system. In the hydrothermal example considered here, the reactions are driven by fluid infiltration along temperature gradients in the convection cell. In other words, the structure of the hydrothermal convection cell, which already is a form of self-organization, causes a geochemical self-organization to develop because of the temperature dependence of the mineral solubilities. In this case, the boundary conditions are important only in specifying the upper and lower temperatures of the convection cell.

Up to about 40,000 years, the geometry of the convection cells becomes increasingly distorted, but the cells still have a wavelength of 4,000 meters. Beginning about 40,000 years, however, the upwelling zones which had initially formed at 0 and 8,000 meters migrate gradually inward until by 50,000 years the plumes are centered at 1400 and 6600 meters. The shift is apparent both from the evolution of the temperature (Figure 23) and the quartz precipitation rate (Figure 22). This behavior indicates that the convection process is fundamentally unstable when reaction-induced permeability changes take place. This is in contrast to the stable behavior of a $\mathrm{Ra}=200$ system in which permeability change does not occur. It also is worth noting the time scales involved. The simulations indicate that the hydrothermal system never attains a steady state when the initial flow porosity is $0.1 \%$. Presumably the transient effects of permeability change are even more extreme where the initial flow porosity is smaller.

\subsection{Implications for Convection in the Crust}

The model presented here for multi-dimensional reactive flow suggests a number of firstorder generalizations about the character of convective flow in the crust, despite the fact that the system considered is highly simplified both chemically and physically. The simulations indicate that within the range of realistic porosity-permeability relations observed in natural settings, it is likely that reaction-induced permeability changes will be important within the life span of crustal hydrothermal systems. The permeability changes occur sufficiently rapidly that the fundamental character of the hydrothermal system is changed. It is unlikely, therefore, that steady state conditions can ever be attained. Moreover, the simulations suggest that the convection process may be fundamentally unstable because of the feedbacks between chemistry and the properties of the rock. The dominant effect which is observed in the simulations is that where fluids moving up or down a temperature gradient drive precipitation or dissolution reactions (or simply overall reactions in which 
there is a net volume change), the convection cell tends to organize itself, or perhaps more appropriately, reorganize itself. In the example considered here, quartz precipitation reduces the permeability of the rock to the point where the upwelling portion of a convection cell becomes increasingly diffuse while quartz dissolution has the opposite effect, causing the flow to become increasingly channelized. In agreement with the results of Ortoleva and others (1987) and Steefel and Lasaga (1990), the dissolution of the rock leads to an instability in which enhanced permeability increases the fluid flux which in turn increases the rates at which the reactions proceed. To our knowledge, this effect has not been demonstrated previously in a nonisothermal setting. It also points out the importance of considering porosity and permeability changes as a multi-dimensional process since the effect does not appear at all in one-dimensional systems (e.g., Wells and Ghiorso, 1991).

The simulations presented here also give some credence to the hypothesis suggested by several workers that the maximum in the solubility of quartz which appears in relatively low pressure hydrothermal systems (e.g., below about $1 \mathrm{kbar}$ ) may result in a permeability barrier below which fluids cannot easily circulate (Truesdell and Fournier; 1976; Bischoff and Rosenbauer, 1985; Wells and Ghiorso, 1991). Bischoff and Rosenbauer (1985) showed that for seawater, quartz shows a maximum in its solubility at temperatures as low as $340^{\circ} \mathrm{C}$ as the critical point of water is approached. Although not explicitly considered here since we have restricted ourselves to temperatures below $300^{\circ} \mathrm{C}$, the simulations imply that recharging waters in a convection cell would tend to leach quartz (and perhaps other silicate minerals) up to the temperatures corresponding to the solubility maximum. A recharging fluid moving beyond this temperature would tend to precipitate quartz, thus reducing the rock permeability further. An interface might develop at a temperature of about $350^{\circ} \mathrm{C}$ separating a low permeability zone at depth from a high permeability zone above. [It is possible, therefore, that the topology of the quartz solubility curve eventually imposes a physical structure on the convection cell by generating its own permeability contrasts.] This process probably does not occur on the short time scales suggested by Wells and Ghiorso (1991), however, since recharging fluids are likely to be moving through rocks of much lower permeability than those characterizing the hydrothermal conduits that they considered. Nontheless, the simulations presented here indicate that the process could become important within about 5,000 to 10,000 years after the onset of vigorous convection (i.e, neglecting the initial startup phase of the convection cell) depending on the permeability of the recharge zone.

Given the substantial changes in the permeability of the rock which the simulations predict are likely to occur over the time scale of tens of thousands of years, it is interesting to speculate on how it is possible to maintain crustal hydrothermal systems for long periods of time. The geothermal system in Yellowstone Park, U.S.A is a minimum of 15,000 years old and probably much closer to the 0.6 Ma during which the Yellowstone caldera has existed (Fournier, 1989) and most of the oceanic hydrothermal systems show evidence of convection for millions of years (e.g., Johnson and Carlson, 1992). One possiblity, of course, is that the chemical reactions involved are such that they involve no net change 
in the volume of the rock. This seems unlikely to be true, however, everywhere within the hydrothermal system. A more likely explanation is that there is some rate at which new fractures are generated which keeps the convection process alive, if not necessarily stable. There is abundant field evidence of numerous generations of hydrothermal veining in both continental and marine settings, so this would seem to be a logical explanation. The origin of the fracturing is less certain, whether due to regional shear stresses or to the infiltration of cold water into hot rock (Lister, 1974; Lister, 1983). If the process is the result of thermal cracking due to the infiltration of cold water, then the behavior of the system may be similar to that shown in the simulations of permeability change since recharge regions would experience a permeability increase while the upwelling zones would undergo a permeability decrease. If this is what occurs, then it seems likely the convection process would still be unstable, periodically reorganizing itsclf because of the changing permeability distribution.

\title{
DISCLAIMER
}

\begin{abstract}
This report was prepared as an account of work sponsored by an agency of the United States Government. Neither the United States Government nor any agency thereof, nor any of their employees, makes any warranty, express or implied, or assumes any legal liability or responsibility for the accuracy, completeness, or usefulness of any information, apparacus, product, or process disclosed, or represents that its use would not infringe privately owned rights. Reference herein to any specific commercial product, process, or service by trade name, trademark, manufacturer, or otherwise does not necessarily constitute or imply its endorsement, recommendation, or favoring by the United States Government or any agency thereof. The views and opinions of authors expressed herein do not necessarily state or reflect those of the United States Government or any agency thereof.
\end{abstract}



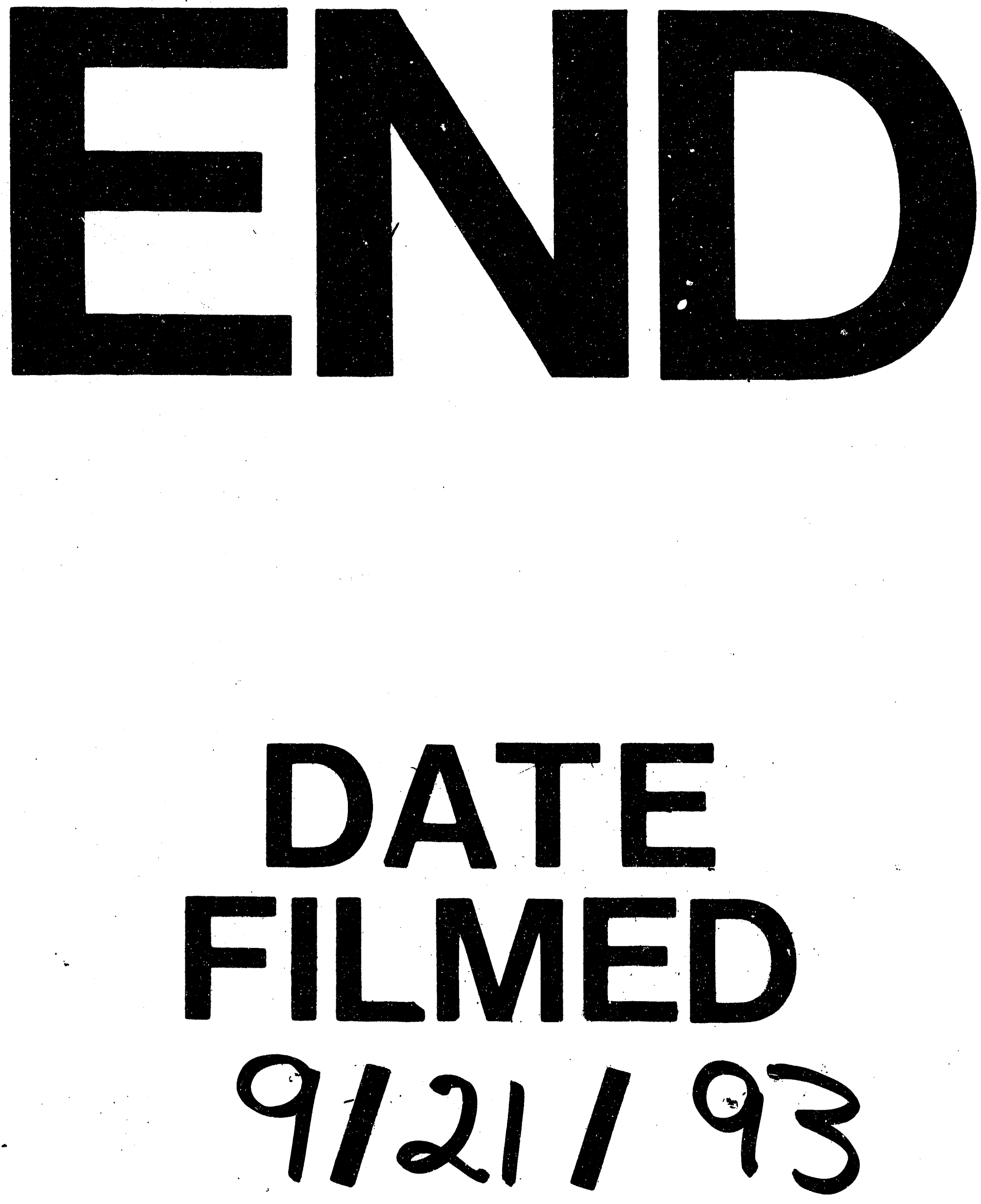